\title{
On the Finite Difference Approximation for a Parabolic Blow-Up Problem
}

\author{
C.-H. Cho, S. Hamada and H. Okamoto
}

Research Institute for Mathematical Sciences

Kyoto University, Kyoto 606-8502, Japan

E-mail: okamoto@kurims.kyoto-u.ac.jp

Received December 16, 2005

Revised October 13, 2006

\section{Introduction}

We consider a scalar semi-linear parabolic partial differential equation $u_{t}=$ $u_{x x}+f(u)(0 \leq t, 0 \leq x \leq 1)$ with the Dirichlet boundary condition $u=0$ on $x=0$ and $x=1$, where $f: \mathbb{R} \rightarrow \mathbb{R}$ is a smooth function. It is known (see, $[4,8,10,11]$ ) that a solution with a large initial data blows up in finite time, if an appropriate growth condition on $f$ as $u \rightarrow \infty$ is imposed. We are concerned in this paper with a question as to how a finite difference scheme can reproduce the blow-up phenomena. By studying various papers, we found that many interesting problems for numerical analysis of the parabolic blow-up problem are left unsolved. And we would like to solve some of them in the present paper.

Let us recall a pioneering paper by Nakagawa [12]. With $f(u)=u^{2}$, Nakagawa [12] considered a finite difference scheme, where a uniform mesh was used for the spatial variable and a certain adaptive mesh was used for the time variable. He then showed that his finite difference solutions blow up in finite time if a certain largeness condition on the initial data is assumed and that the numerical blow-up time converges to the 'real' blow-up time if the mesh size tends to zero. Let $T>0$ be the blow-up time. Then it is not difficult to prove that the finite difference scheme converges in $0 \leq t \leq \tilde{T}$ for any prescribed $\tilde{T}<T$. This is a classical result, but Nakagawa did much more. His result is important in that he proved the convergence up to the blow-up time. Later, a finite element analogue was considered by Nakagawa and Ushijima [13]. Quite general blow-up conditions were established for semi-discretized equations by T.K. Ushijima [16]. See also [1, 2]. Recently a substantial generalization was made on the convergence of the blow-up time by Abia and others [3]. A different approach was proposed by Hirota and Ozawa [9].

A new direction was explored by Chen [6], who considered the equation with $f(u)=u^{1+\alpha}$ and proved, among others, that some numerical solutions can blow up at more than one point, while a one-point blow-up is known to occur in the continuous problem. Thus, a finite difference scheme with a spatially uniform mesh does not correctly reproduce the blow-up phenomena. Correct meaning will be stated later. 
The purpose of the present paper is to provide clues to those problems which were left unanswered or untouched in these papers.

More sophisticated schemes than are studied in these papers have been proposed by many researchers, see for instance $[14,16]$ and the references therein. For practical computations of blow-up phenomena, such schemes with spatially adaptive meshes are highly recommended, but they are, at the same time, hardly amenable to rigorous convergence proof. We are interested in those results in which mathematically rigorous analysis can be carried out. In this reason, we focus our attention to the simplest case. Tabata's scheme in [15] is rather simple and at the same time very useful in improving the stability, but, for the same reason, we do not consider it, either.

Although the details are explained in the subsequent sections, we here outline what we are going to study: Let us recall a result of Nakagawa [12]. He considered $u_{t}=u_{x x}+u^{2}$ in $0 \leq x \leq 1$ with the Dirichlet boundary condition $u=0$ on $x=0,1$. He then divided $[0,1]$ into $N$ subintervals of equal length. With $h=1 / N$ we define the discrete $L^{p}$-norm $(1 \leq p<\infty)$ for $w=\left(w_{1}, w_{2}, \ldots, w_{N-1}\right)$ as

$$
\|w\|_{p}=\left\{h \sum_{k=1}^{N-1}\left|w_{k}\right|^{p}\right\}^{1 / p} .
$$

We also define

$$
\|w\|_{\infty}=\max _{1 \leq k \leq N-1}\left|w_{k}\right| .
$$

Nakagawa considered the following finite difference scheme:

$$
\begin{aligned}
& \frac{u_{j}^{n+1}-u_{j}^{n}}{\Delta t_{n}}=\frac{u_{j+1}^{n}-2 u_{j}^{n}+u_{j-1}^{n}}{h^{2}}+\left(u_{j}^{n}\right)^{2} \quad\left(\begin{array}{l}
n=0,1, \ldots, \\
j=1,2, \ldots, N-1
\end{array}\right), \\
& \Delta t_{n}=\tau \min \left\{1, \frac{1}{\left\|u^{n}\right\|_{2}}\right\},
\end{aligned}
$$

where $\tau$ is a prescribed positive parameter, $u_{j}^{n}$ is an approximation for $u\left(t_{n}, j h\right)$, $u^{n}=\left(u_{1}^{n}, u_{2}^{n}, \ldots, u_{N-1}^{n}\right)$, and $\left\|u^{n}\right\|_{2}$ denotes the discrete $L^{2}$-norm. Nodal points $t_{n}$ are defined as $t_{n}=\sum_{j=0}^{n-1} \Delta t_{j}, t_{0}=0$. The boundary condition $u_{0}^{n}=u_{N}^{n}=0$ is imposed. Here, by the rule (2) he intends that the uniform mesh $\Delta t_{n}=\tau$ is employed while the data is so small that $\left\|u^{n}\right\|_{2} \leq 1$ and that an adaptive mesh is used in the case where $u^{n}$ is so large that $\left\|u^{n}\right\|_{2}>1$. The numerical blow-up time is defined as

$$
T(\tau, h)=\sum_{j=0}^{\infty} \Delta t_{j}=\lim _{n \rightarrow \infty} t_{n} .
$$

He proved under a certain stability condition that $T(\tau, h)$ tends to $T$, the blow-up time of $u$, as $\tau \downarrow 0$ and $h \downarrow 0$. We remark, however, that he did not derive a convergence rate of $|T(\tau, h)-T|$. 
The following questions may naturally arise:

1. Can we have an error estimate such as $|T-T(\tau, h)|=O\left(\tau^{s}\right)$ with some positive $s>0$ ?

2. Why did he choose the definition of $\Delta t_{n}$ as in (2)? Can we equally use

$$
\Delta t_{n}=\tau \min \left\{1, \frac{1}{\left\|u^{n}\right\|_{p}^{\gamma}}\right\}
$$

where $p \in[1, \infty]$ and $\gamma>0$ is a positive constant different from 1.

We wanted to answer these questions but we found that the question 1 was rather difficult. Accordingly, what we can answer in the subsequent sections is partial. On the other hand, the question 2 can be better answered. In fact, Abia et al. [3] proved the convergence in the case where $L^{\infty}$-norm was used and $\gamma=1$. We prove in the next section that not only general $p$ but also $\gamma$ 's of some range can be used in the definition of $\Delta t_{n}$ without destroying the convergence.

As we will show soon, our knowledge on the convergence rate for partial differential equations (PDEs) is quite incomplete. On the other hand, we can prove a rather complete theorem for a scalar ordinary differential equation (ODE). We therefore devote one section for scalar ODEs. The result for ODE is also important in its vital role when we consider the convergence for PDE.

REMARK 1.1. When we actually compute, it is better to define $\Delta t_{n}$ as

$$
\Delta t_{n}=\tau \min \left\{c_{0}, \frac{1}{\left\|u^{n}\right\|_{2}}\right\}
$$

with another constant $c_{0}$ than to define it by (2). But this is unimportant in the subsequent mathematical analysis, and we always set $c_{0}=1$.

Next, let us recall a result of Chen [12]. He considered $u_{t}=u_{x x}+u^{1+\alpha}$ in $0 \leq x \leq 1$ mainly with the Neumann boundary condition $u_{x}=0$ on $x=0,1$ and the initial data $u_{0}(x)$ which satisfies the following two conditions:

(i) $\quad u_{0}(x)$ is symmetric about $x=1 / 2$;

(ii) $u_{0}(x)$ is monotone increasing in $[0,1 / 2]$.

He then considered a Nakagawa-like scheme with

$$
\Delta t_{n}=\tau \min \left\{1, \frac{1}{\left\|u^{n}\right\|_{1}^{\alpha}}\right\} .
$$

We now make the following definition:

Definition 1.1. If $\lim _{n \rightarrow \infty} u_{j}^{n}=\infty$, we call $x_{j}$ a numerical blow-up point. The set of all numerical blow-up points is called the numerical blow-up set.

Chen showed that when $\alpha>1$, the only numerical blow-up point is the mid-point $x=1 / 2$, and that the finite difference solutions blow up at more than one point if $\alpha \leq 1$. This is an interesting phenomena in view of the fact that the solutions of the corresponding PDE blow up only at one point $x=1 / 2$ for any $0<\alpha<\infty$. 
After having read his paper, the following questions naturally occurred to us.

1. Although he proved that the numerical solutions blow up at exactly three point if $\alpha=1$ and at least at three points if $0<\alpha<1$, the blow-up set was not completely determined in the case of $0<\alpha<1$. Can we determine the blow-up set exactly?

2. What happens in the case of other nonlinear terms, say, e.g., $f(u)=$ $u^{1+\alpha}[\log (1+u)]^{\beta}$ with a positive constant $\beta$ ?

As we will show soon, we can determine the numerical blow-up set for the above nonlinear term. Further, we can prove an interesting phenomena in the case of $f(u)=u\{\log (1+u)\}^{\beta}$ that there is an unsurmountable discrepancy between the numerical blow-up set and the blow-up set of the corresponding PDE.

\section{Scalar ODE}

We consider in this section a scalar ODE $\dot{u}=G(u)$ and its finite difference approximation. Throughout this section, we impose the following assumptions on $G$;

1. $G:[0, \infty) \rightarrow[0, \infty)$ is continuously differentiable;

2. $G(s)>0$ for $s>0$;

3. $G$ is strictly monotone increasing;

4. $\int_{1}^{\infty} G(s)^{-1} \mathrm{~d} s<\infty$.

Also we consider only those solutions with $u(0)>0$. Our assumptions imply that any solution of $\dot{u}=G(u)$ with $u(0)>0$ is increasing and convex in $t$ and that it blows up in finite time. The blow-up time of $u$ is obviously $\int_{u(0)}^{\infty} G(s)^{-1} \mathrm{~d} s$, which will be denoted by $T$.

Let us consider the following finite difference scheme:

$$
\frac{v^{n+1}-v^{n}}{\Delta t_{n}}=G\left(v^{n}\right) \quad(n=0,1,2, \ldots)
$$

where we assume that $v^{0}=u(0)>0$, and $\Delta t_{n}$ is defined by

$$
\Delta t_{n}=\tau \min \left\{1, \frac{1}{H\left(v^{n}\right)}\right\} .
$$

Here $H=H(s)$ is another function which is assumed to be positive for positive $s$, to be monotone increasing, and to satisfy $\lim _{s \rightarrow \infty} H(s)=+\infty . \tau>0$ is a parameter. For later use we define $t_{0}=0$ and $t_{n}=\sum_{j=0}^{n-1} \Delta t_{j}$. We now define the 'numerical blow-up time':

Definition 2.1. We define

$$
T(\tau)=\sum_{n=0}^{\infty} \Delta t_{n}=\lim _{n \rightarrow \infty} t_{n}
$$

and call it the numerical blow-up time. 
At this stage the possibility that $T(\tau)=\infty$ is not excluded. But we can prove that it is finite under certain assumptions on $G$ and $H$.

THEOREM 2.1. In addition to the assumptions above, we assume that

$$
z \mapsto z+\tau \frac{G(z)}{H(z)}
$$

is monotone increasing in $H^{-1}(1)<z<\infty$. We also assume that

$$
\int_{H^{-1}(1)}^{\infty} \frac{G^{\prime}(z)}{G(z) H(z)} \mathrm{d} z<\infty .
$$

We then have

$$
T<T(\tau)<T+c \tau,
$$

where $c$ is a positive constant depending only on $G$ and $u(0)$.

Proof. We first show that $H\left(v^{j}\right)>1$ for all sufficiently large $j$. To this end, we note first that $\left\{v^{n}\right\}$ is monotone increasing. It therefore tends to a limit $v^{\infty} \leq \infty$. We are done if $v^{\infty}>H^{-1}(1)$. Let us therefore assume on the contrary that $v^{\infty} \leq H^{-1}(1)(<\infty)$. But this implies that $\Delta t_{n}=\tau$ for all $n$, which together with (3) gives us $v^{\infty}=v^{\infty}+\tau G\left(v^{\infty}\right)$. This, however, cannot hold because of our positivity assumption on $G$. We accordingly have

$$
v^{n+1}=v^{n}+\tau \frac{G\left(v^{n}\right)}{H\left(v^{n}\right)}
$$

for sufficiently large n's. We now repeat the same argument to obtain

$$
\lim _{n \rightarrow \infty} v^{n}=+\infty
$$

We now prove that $T<T(\tau)$. To see this, we note that

$$
T=\int_{u(0)}^{\infty} \frac{\mathrm{d} s}{G(s)}
$$

and

$$
T(\tau)=\sum_{j=0}^{\infty} \Delta t_{j}=\sum_{j=0}^{\infty} \frac{v^{j+1}-v^{j}}{G\left(v^{j}\right)} .
$$

We then have $T<T(\tau)$ by $(8), v^{0}=u(0)$, and Fig. 1 .

In order to prove the other inequality, we take the non-negative integer $m$ such that $\Delta t_{j}=\tau / H\left(v^{j}\right)$ for all $j \geq m$ and that $\Delta t_{j}=\tau$ for $j<m$. We then define a function of $y$ as follows:

$$
\phi(y)=G^{-1}\left(\frac{1}{y}\right)+\frac{\tau}{y H\left(G^{-1}(1 / y)\right)} \quad(0<y<\infty),
$$




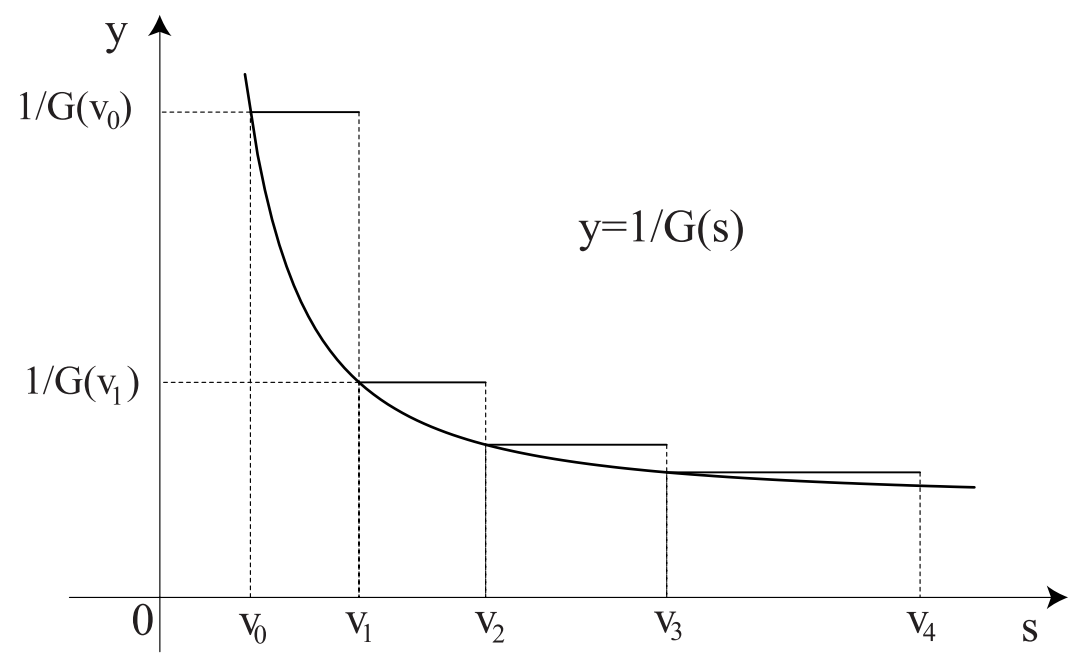

Fig. 1.

where $G^{-1}$ denotes the inverse function (not $1 / G(s)$ ). Note that this function satisfies $\phi\left(1 / G\left(v^{j}\right)\right)=v^{j+1}$ for all $j \geq m$. Note also that the assumption (5) with a substitution $z=G^{-1}(1 / y)$ implies that $\phi$ is monotone decreasing in $0<y<$ $1 / G\left(v^{m}\right)$. With these observations, we see from Fig. 2 that

$$
\begin{aligned}
\sum_{j=m}^{n-1} \frac{v^{j+1}-v^{j}}{G\left(v^{j}\right)} \leq & \int_{1 / G\left(v^{n-1}\right)}^{1 / G\left(v^{m}\right)} \phi(y) \mathrm{d} y-v^{m}\left(\frac{1}{G\left(v^{m}\right)}-\frac{1}{G\left(v^{n-1}\right)}\right)+\frac{v^{n}-v^{m}}{G\left(v^{n-1}\right)} \\
= & \int_{v^{m}}^{v^{n-1}}\left[z+\frac{\tau G(z)}{H(z)}\right] \frac{G^{\prime}(z)}{G(z)^{2}} \mathrm{~d} z+\frac{v^{n}}{G\left(v^{n-1}\right)}-\frac{v^{m}}{G\left(v^{m}\right)} \\
= & -\left.\frac{z}{G(z)}\right|_{v^{m}} ^{v^{n-1}}+\int_{v^{m}}^{v^{n-1}} \frac{\mathrm{d} z}{G(z)}+\tau \int_{v^{m}}^{v^{n-1}} \frac{G^{\prime}(z) \mathrm{d} z}{G(z) H(z)} \\
& +\frac{v^{n}}{G\left(v^{n-1}\right)}-\frac{v^{m}}{G\left(v^{m}\right)} \\
= & \int_{v^{m}}^{v^{n-1}} \frac{\mathrm{d} z}{G(z)}+\tau \int_{v^{m}}^{v^{n-1}} \frac{G^{\prime}(z) \mathrm{d} z}{G(z) H(z)}+\frac{v^{n}-v^{n-1}}{G\left(v^{n-1}\right)} \\
= & \int_{v^{m}}^{v^{n-1}} \frac{\mathrm{d} z}{G(z)}+\tau \int_{v^{m}}^{v^{n-1}} \frac{G^{\prime}(z) \mathrm{d} z}{G(z) H(z)}+\frac{\tau}{H\left(v^{n-1}\right)}
\end{aligned}
$$

We therefore have

$$
t_{n}-t_{m} \leq \int_{v^{m}}^{v^{n-1}} \frac{\mathrm{d} z}{G(z)}+\tau \int_{v^{m}}^{v^{n-1}} \frac{G^{\prime}(z)}{G(z) H(z)} \mathrm{d} z+\frac{\tau}{H\left(v^{n-1}\right)}
$$




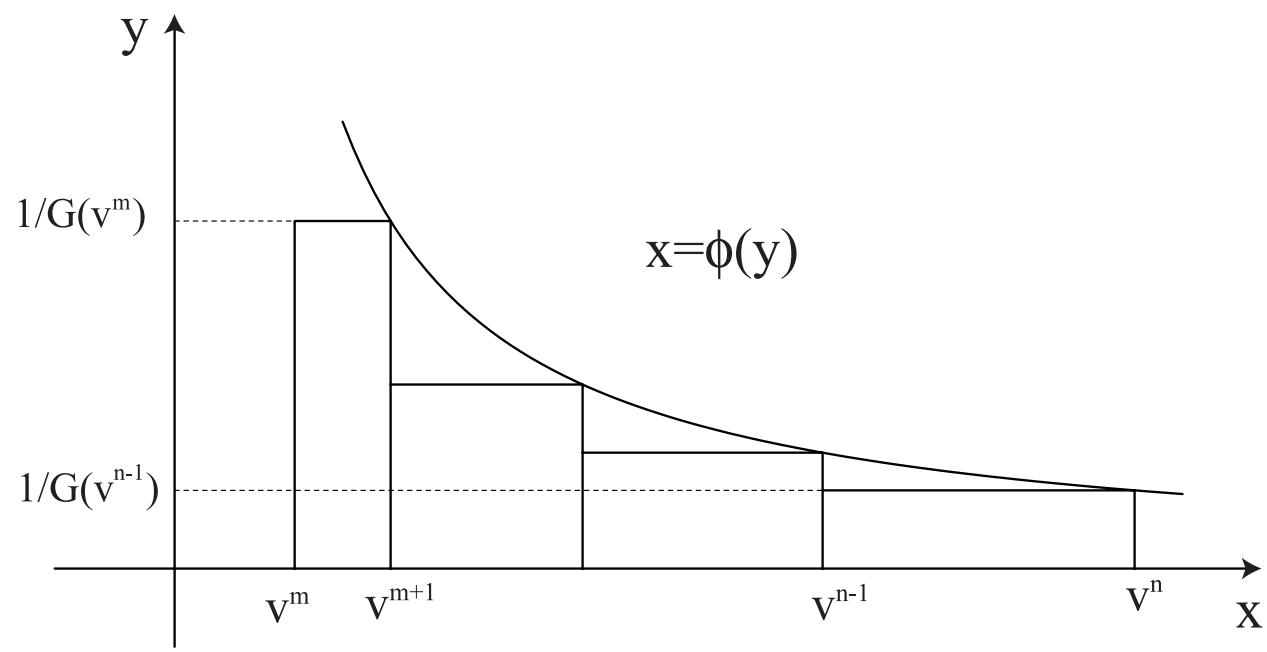

Fig. 2 .

We next note that the solution $u(t)$ of $\dot{u}=G(u)$ is increasing and convex in $t$. Then

$$
u\left(t_{1}\right)=u(0)+\int_{0}^{t_{1}} G(u(s)) \mathrm{d} s>u(0)+t_{1} G(u(0))=v^{1} .
$$

It is then easy to prove by induction that

$$
u\left(t_{n}\right) \geq v^{n} .
$$

(This is true whatever the definition of $\Delta t_{n}$ may be.)

A classical result on the convergence of finite difference schemes says that a finite difference solution converges with an order $O(\tau)$ to the solution of ODE, so long as the solution is smooth. This fact and (10) imply that $u\left(t_{m}\right)-c \tau \leq$ $v^{m} \leq u\left(t_{m}\right)$, where $c$ is a positive constant depending only on $G$ and $u_{0}$. Since $t_{m}=\int_{u(0)}^{u\left(t_{m}\right)} \frac{\mathrm{d} s}{G(s)}$, we have

$$
0<t_{m}-\int_{u(0)}^{v^{m}} \frac{\mathrm{d} s}{G(s)}<c^{\prime} \tau
$$

where $c^{\prime}$ is another positive constant. Combining this with (9), we obtain

$$
t_{n} \leq \int_{u(0)}^{v^{n-1}} \frac{\mathrm{d} z}{G(z)}+\tau \int_{v^{m}}^{v^{n-1}} \frac{G^{\prime}(z)}{G(z) H(z)} \mathrm{d} z+\frac{\tau}{H\left(v^{n-1}\right)}+c^{\prime} \tau,
$$

which implies that

$$
\begin{aligned}
T(\tau) & \leq \int_{u(0)}^{\infty} \frac{\mathrm{d} z}{G(z)}+\tau \int_{v^{m}}^{\infty} \frac{G^{\prime}(z)}{G(z) H(z)} \mathrm{d} z+c^{\prime} \tau \\
& \leq T+\left(\int_{H^{-1}(1)}^{\infty} \frac{G^{\prime}(z)}{G(z) H(z)} \mathrm{d} z+c^{\prime}\right) \tau .
\end{aligned}
$$


Therefore we are done.

Corollary 2.1. For all $l \geq m$, it holds that

$$
T(\tau) \leq t_{l}+\int_{v^{l}}^{\infty} \frac{\mathrm{d} z}{G(z)}+\tau \int_{v^{l}}^{\infty} \frac{G^{\prime}(z)}{G(z) H(z)} \mathrm{d} z .
$$

The proof is obvious if we note that (9) holds true if we replace $m$ with $l$.

Corollary 2.2. Suppose that $G(u)=u^{1+\alpha}$ and $H(s)=s^{\gamma}$, where $\alpha>0$ and $\gamma>0$ are positive constants. We then have $T<T(\tau)<T+c \tau$ if $0<\gamma \leq$ $1+\alpha+\tau^{-1}$. Also,

$$
0<T(\tau)-T<\frac{(1+\alpha) \tau}{\gamma\left(v^{m}\right)^{\gamma}}+c^{\prime} \tau \leq \frac{(1+\alpha) \tau}{\gamma}+c^{\prime} \tau
$$

Proof. In order to verify the monotonicity (5), we note that $z+\tau G(z) / H(z)=$ $z+\tau z^{1+\alpha-\gamma}$, whose derivative is $1+\tau(1+\alpha-\gamma) z^{\alpha-\gamma}$. This is positive for all $z>1=H^{-1}(1)$ if $1+\tau(1+\alpha-\gamma) \geq 0$, i.e., $\gamma \leq 1+\alpha+\tau^{-1}$. We easily see that

$$
\int_{v^{m}}^{\infty} \frac{G^{\prime}(z)}{G(z) H(z)} \mathrm{d} z=\frac{(1+\alpha) \tau}{\gamma\left(v^{m}\right)^{\gamma}} .
$$

REMARK 2.1. In view of (12), one might be tempted to conclude that the larger $\gamma$ is, the more accurate the numerical blow-up time becomes; hence, $\gamma=$ $1+\alpha+\tau^{-1}$ is the best choice. However, this is not the case. For, first, the estimate (12) is only an upper bound. Second, we should, at the same time, worry about the number of computer operations required. In fact, the larger $\gamma$ is, the more computer operations are required (since $\Delta t_{n}$ becomes smaller). Accordingly, a large $\gamma$ is not necessarily a good choice. We will come back to this issue at the end of the next section.

\section{A generalization of Nakagawa's result}

In this section, we consider a PDE

$$
\frac{\partial u}{\partial t}=\frac{\partial^{2} u}{\partial x^{2}}+f(u) \quad(0 \leq t, 0 \leq x \leq 1)
$$

with the Dirichlet boundary condition

$$
u(t, 0)=u(t, 1)=0
$$

and an initial condition

$$
u(0, x)=u_{0}(x) .
$$

We assume that the initial data $u_{0}(x)$ is non-negative everywhere in $[0,1]$. We impose the following assumptions on $f:[0, \infty) \rightarrow[0, \infty)$; 
1. $f$ is continuously differentiable;

2. $f(s)>0$ for $s>0$;

3. $f$ is strictly monotone increasing and convex;

4. $\int_{1}^{\infty} f(s)^{-1} d s<\infty$.

These assumptions imply that for a 'large' initial data $u_{0}(x)$ the solution of (13) (14) (15) blows up in finite time, see e.g., [4, 8, 10, 17]. We denote the blow-up time of $u$ by $T$. Before entering the main contents, we prove the following:

Lemma 3.1. For any $b \in(0,1)$ and any $K>0$, there exists a constant $s_{0}>0$ such that

$$
b f(s)+K s \leq f(s)
$$

holds true for all $s \geq s_{0}$.

Proof. The convexity of $f$ implies that $f^{\prime}$ is increasing. We actually have $\lim _{s \rightarrow \infty} f^{\prime}(s)=\infty$. In fact, if $f^{\prime}$ is bounded, say $f^{\prime}(s) \leq c$, then we have $f(s) \leq$ $c s+c^{\prime}$ with a constant $c^{\prime}$. But this contradicts the assumption 4. Accordingly, there exists $s_{1}$ such that

$$
\frac{2 K}{1-b} \leq f^{\prime}(s) \quad\left(s_{1} \leq s\right)
$$

The integration gives us

$$
f\left(s_{1}\right)-\frac{2 K s_{1}}{1-b}+\frac{2 K s}{1-b} \leq f(s) .
$$

On the other hand, if we choose an $s_{0} \geq s_{1}$ such that

$$
0 \leq f\left(s_{1}\right)-\frac{2 K s_{1}}{1-b}+\frac{K s_{0}}{1-b},
$$

then, it holds, for all $s \geq s_{0}$, that $f(s) \geq K s /(1-b)$, which is to be shown.

Let us consider the following finite difference scheme:

$$
\frac{u_{j}^{n+1}-u_{j}^{n}}{\Delta t_{n}}=\frac{u_{j+1}^{n}-2 u_{j}^{n}+u_{j-1}^{n}}{h^{2}}+f\left(u_{j}^{n}\right),
$$

with the following boundary condition

$$
u_{0}^{n}=u_{N}^{n}=0 \quad(n=0,1,2, \ldots)
$$

and the initial condition

$$
u_{j}^{0}=u_{0}\left(x_{j}\right) \quad(j=1, \ldots, N-1),
$$

where $h=1 / N$, and $x_{j}=j h$ are spatial grid points $(j=0,1, \ldots, N)$. We define $\Delta t_{n}$ by

$$
\Delta t_{n}=\tau \min \left\{1, \frac{1}{g\left(\left\|u^{n}\right\|_{p}\right)}\right\}
$$


Here, $1 \leq p \leq \infty$, and $g:[0, \infty) \rightarrow[0, \infty)$ is a function which satisfies certain properties specified below. Note that $\Delta t_{n} \leq \tau$ for all $u^{n}$. We also define $t_{n}$ by

$$
t_{0}=0, \quad t_{n}=\sum_{i=0}^{n-1} \Delta t_{i}
$$

and the numerical blow-up time of (16) (17) (18) by

$$
T(\tau, h)=\lim _{n \rightarrow \infty} t_{n}=\sum_{i=0}^{\infty} \Delta t_{i} .
$$

Before studying the blow-up of $\left\{u_{j}^{n}\right\}$, we prove that all the solutions of (16) converge to the corresponding solution of (13) as far as the smoothness of $u$ is guaranteed.

TheOREM 3.1. Let $\left\{u_{j}^{n}\right\}$ be the solution of (16) (17) (18). Let $\lambda$ be defined by $\lambda=\tau / h^{2}$ and assume that $0<\lambda \leq 1 / 2$. Let $T$ denote the blow-up time of $u$ and let $T_{0}$ be an arbitrary number such that $0<T_{0}<T$. Then there exists positive constants $C$ and $h_{0}$, depending only on $T_{0}$ and $u_{0}$, such that

$$
\max _{1 \leq j \leq N-1}\left|u_{j}^{n}-u\left(t_{n}, x_{j}\right)\right| \leq C h^{2}
$$

holds so far as $t_{n} \leq T_{0}$ and $0<h \leq h_{0}$.

Proof. Although this theorem is essentially proved in Nakagawa [12], we outline a proof for reader's convenience and for the reason that our scheme is more general than his.

Let $\varepsilon_{j}^{n}=u_{j}^{n}-u\left(t_{n}, x_{j}\right)$. By the Taylor expansion theorem, we have

$$
\frac{u\left(t_{n+1}, x_{j}\right)-u\left(t_{n}, x_{j}\right)}{\Delta t_{n}}=\frac{\partial u}{\partial t}\left(t_{n}, x_{j}\right)+\frac{\Delta t_{n}}{2} \frac{\partial^{2} u}{\partial t^{2}}\left(t_{n}+\theta_{1} \Delta t_{n}, x_{j}\right)
$$

and

$$
\begin{aligned}
& \frac{u\left(t_{n}, x_{j+1}\right)-2 u\left(t_{n}, x_{j}\right)+u\left(t_{n}, x_{j-1}\right)}{h^{2}} \\
& =\frac{\partial^{2} u}{\partial x^{2}}\left(t_{n}, x_{j}\right)+\frac{h^{2}}{24}\left\{\frac{\partial^{4} u}{\partial x^{4}}\left(t_{n}, x_{j}+\theta_{2} h\right)+\frac{\partial^{4} u}{\partial x^{4}}\left(t_{n}, x_{j}-\theta_{3} h\right)\right\},
\end{aligned}
$$

where $0 \leq \theta_{i} \leq 1(i=1,2,3)$. Also, by the mean-value theorem,

$$
f\left(u_{j}^{n}\right)-f\left(u\left(t_{n}, x_{j}\right)\right)=f^{\prime}\left(u\left(t_{n}, x_{j}\right)+\theta_{4} \varepsilon_{j}^{n}\right) \cdot \varepsilon_{j}^{n}
$$

for some $\theta_{4} \in[0,1]$. These equations are combined to yield

$$
\frac{\varepsilon_{j}^{n+1}-\varepsilon_{j}^{n}}{\Delta t_{n}}=\frac{\varepsilon_{j+1}^{n}-2 \varepsilon_{j}^{n}+\varepsilon_{j-1}^{n}}{h^{2}}+f^{\prime}\left(u\left(t_{n}, x_{j}\right)+\theta_{4} \varepsilon_{j}^{n}\right) \varepsilon_{j}^{n}+r_{j}^{n},
$$


where

$$
r_{j}^{n}=-\frac{\Delta t_{n}}{2} \frac{\partial^{2} u}{\partial t^{2}}\left(t_{n}+\theta_{1} \tau_{n}, x_{j}\right)+\frac{h^{2}}{24}\left\{\frac{\partial^{4} u}{\partial x^{4}}\left(t_{n}, x_{j}+\theta_{2} h\right)+\frac{\partial^{4} u}{\partial x^{4}}\left(t_{n}, x_{j}+\theta_{3} h\right)\right\} .
$$

Put

$$
\begin{aligned}
E^{n} & =\max _{1 \leq j \leq N-1}\left|\varepsilon_{j}^{n}\right|, \quad U=\max _{(t, x) \in\left[0, T_{0}\right] \times[0,1]}|u(t, x)|, \quad K=f^{\prime}(U+1), \\
R & =\frac{\lambda}{2} \max _{\substack{0 \leq t \leq T_{0} \\
0 \leq x \leq 1}}\left|\frac{\partial^{2} u}{\partial t^{2}}(t, x)\right|+\frac{1}{12} \max _{\substack{0 \leq t \leq T_{0} \\
0 \leq x \leq 1}}\left|\frac{\partial^{4} u}{\partial x^{4}}(t, x)\right| .
\end{aligned}
$$

Then, as far as $E^{n} \leq 1$, (19) gives us $E^{n+1} \leq\left(1+K \Delta t_{n}\right) E^{n}+h^{2} R \Delta t_{n}$. From this inequality, we obtain

$$
E^{n+1} \leq e^{K T_{0}}\left(E^{0}+h^{2} R T_{0}\right)=e^{K T_{0}} h^{2} R T_{0}
$$

If $h$ is sufficiently small, then the right hand side of (20) is smaller than 1 . Hence (20) holds true for all $n$.

From now we consider the blow-up of (16). Our goal in this section is to generalize Nakagawa's convergence theorem in the following way.

TheOREM 3.2. Suppose that $g:[0, \infty) \rightarrow[0, \infty)$ satisfies

(i) $g(s)>0$ for $s>0$;

(ii) $g$ is monotone increasing;

(iii) $g(s) \rightarrow \infty$ as $s \rightarrow \infty$;

(iv) the function

$$
z \mapsto z+\tau \frac{f(z)}{g(z)}
$$

is monotone increasing in $g^{-1}(1)<z<\infty$. We also assume that

$$
\int_{g^{-1}(1)}^{\infty} \frac{f^{\prime}(s)}{f(s) g(s)} d s<\infty .
$$

We finally assume that $\tau / h^{2} \leq 1 / 2$. Then we have

$$
T(\tau, h)<\infty \quad \text { and } \quad \lim _{h \rightarrow 0} T(\tau, h)=T .
$$

REMARK 3.1. Since $\tau / h^{2} \leq 1 / 2, h \rightarrow 0$ implies that $\tau \rightarrow 0$. However, as far as $\tau / h^{2} \leq 1 / 2$ is satisfied, $\tau$ 's approach to zero is arbitrary.

REMARK 3.2. Nakagawa [12] proved this theorem in a special case where $f(u)=u^{2}, g(s)=s, p=2$. Chen [6] proved a similar theorem in a special case where $f(u)=u^{1+\alpha}, g(s)=s^{\alpha}, p=1$. A generalization of these theorems is given in [3] in the case of $p=\infty$ with a particular $g$. 
We first recall Lemma 3.1, which we use with $b=1 / 2$ and $K=\pi^{2}$ to obtain

$$
\pi^{2} s+\frac{1}{2} f(s) \leq f(s) \quad\left(s_{0} \leq s\right)
$$

Let $\left\{u_{j}^{n}\right\}$ be a solution of (16) (17) (18). By analogy with a classical functional $\frac{\pi}{2} \int_{0}^{1} u(t, x) \sin (\pi x) \mathrm{d} x$, we define a discrete functional $I_{h}[\cdot]$ by

$$
I_{h}\left[u^{n}\right]=\frac{1}{\sigma(h)} \sum_{j=1}^{N-1} h u_{j}^{n} \sin \left(\pi x_{j}\right)
$$

where $h=1 / N, x_{j}=j h$ and $\sigma(h)=h \sum_{j=1}^{N-1} \sin \left(\pi x_{j}\right)$. Since $\sigma(h)$ is the trapezoidal approximation to $\int_{0}^{1} \sin (\pi x) \mathrm{d} x$, the following inequality holds true:

$$
\frac{2}{\pi}-\frac{\pi}{4} h^{2} \leq \sigma(h) \leq \frac{2}{\pi}
$$

(See, for instance, [7]. The right hand side is true $\operatorname{since} \sin (\pi x)$ is concave in $0 \leq x \leq 1$.) We remark that there exists a positive constant $c_{0}$ such that for all $p \in[1, \infty]$ and sufficiently small $h$,

$$
\left\|u^{n}\right\|_{p} \geq c_{0} I_{h}\left[u^{n}\right] .
$$

In fact, it holds that

$$
\begin{aligned}
I_{h}\left[u^{n}\right] & =\frac{1}{\sigma(h)} \sum_{k=1}^{N-1} h u_{k}^{n} \sin \left(\pi x_{k}\right) \leq \frac{1}{\frac{2}{\pi}-\frac{\pi}{4} h^{2}} \sum_{k=1}^{N-1} h u_{k}^{n} \\
& =\frac{1}{\frac{2}{\pi}-\frac{\pi}{4} h^{2}}\left\|u^{n}\right\|_{1} \leq \frac{1}{\frac{2}{\pi}-\frac{\pi}{4} h^{2}}\left\|u^{n}\right\|_{p} .
\end{aligned}
$$

Accordingly we may take $c_{0}=1 / \pi, h_{0}=2 / \pi$. Then (25) holds true for $0<h<h_{0}$.

For the proof of Theorem 3.2, we need the following

LEMMA 3.2. If $u_{0}(x)$ is chosen large enough in such a way that

$$
2 s_{0}<\frac{\pi}{2} \int_{0}^{1} u_{0}(x) \sin (\pi x) \mathrm{d} x
$$

where $s_{0}$ is the number appearing in (24), then it holds for all $n$ and sufficiently small $h$ that

$$
\frac{I_{h}\left[u^{n+1}\right]-I_{h}\left[u^{n}\right]}{\Delta t_{n}} \geq \frac{1}{2} f\left(I_{h}\left[u^{n}\right]\right) .
$$

Proof of Lemma 3.2. We first note that

$$
I_{h}\left[u_{0}\right] \rightarrow \frac{\pi}{2} \int_{0}^{1} u_{0}(x) \sin (\pi x) d x \quad \text { as } \quad h \rightarrow 0 .
$$


So if $u_{0}(x)$ satisfies the condition (26), then we may assume for all sufficiently small $h$ that $s_{0}<I_{h}\left[u_{0}\right]$.

Note next that

$$
\begin{aligned}
\frac{I_{h}\left[u^{n+1}\right]-I_{h}\left[u^{n}\right]}{\Delta t_{n}} & =\frac{1}{\sigma(h)} \sum_{k=1}^{N-1} h \sin \left(\pi x_{k}\right) \frac{u_{k}^{n+1}-u_{k}^{n}}{\Delta t_{n}} \\
& =\frac{1}{\sigma(h)} \sum_{k=1}^{N-1} h \sin \left(\pi x_{k}\right)\left\{\frac{u_{k+1}^{n}-2 u_{k}^{n}+u_{k-1}^{n}}{h^{2}}+f\left(u_{k}^{n}\right)\right\} .
\end{aligned}
$$

We then compute in a way similar to integration by parts as follows:

$$
\begin{aligned}
& h \sum_{k=1}^{N-1} \frac{1}{h^{2}}\left(u_{k+1}^{n}-2 u_{k}^{n}+u_{k-1}^{n}\right) \sin \left(\pi x_{k}\right) \\
= & \sum_{k=1}^{N-1} \frac{1}{h}\left(u_{k+1}^{n}-u_{k}^{n}\right) \sin \left(\pi x_{k}\right)-\sum_{k=0}^{N-2} \frac{1}{h}\left(u_{k+1}^{n}-u_{k}^{n}\right) \sin \left(\pi x_{k+1}\right) \\
= & \sum_{k=0}^{N-1} \frac{1}{h}\left(u_{k+1}^{n}-u_{k}^{n}\right)\left[\sin \left(\pi x_{k}\right)-\sin \left(\pi x_{k+1}\right)\right] \\
= & \sum_{k=1}^{N-1} \frac{u_{k}^{n}}{h}\left[\sin \left(\pi x_{k-1}\right)-2 \sin \left(\pi x_{k}\right)+\sin \left(\pi x_{k+1}\right)\right] \\
= & -4 \sin ^{2}\left(\frac{\pi h}{2}\right) \sum_{k=1}^{N-1} \frac{u_{k}^{n}}{h} \sin \pi x_{k} \geq-\pi^{2} \sigma(h) I_{h}\left[u^{n}\right] .
\end{aligned}
$$

On the other hand, we have by Jensen's inequality,

$$
\frac{1}{\sigma(h)} \sum_{k=1}^{N-1} h \sin \left(\pi x_{k}\right) f\left(u_{k}^{n}\right) \geq f\left(I_{h}\left[u^{n}\right]\right)
$$

Hence, we obtain

$$
\frac{I_{h}\left[u^{n+1}\right]-I_{h}\left[u^{n}\right]}{\Delta t_{n}} \geq-\pi^{2} I_{h}\left[u^{n}\right]+f\left(I_{h}\left[u^{n}\right]\right) .
$$

Since $I_{h}\left[u^{0}\right] \geq s_{0}$, it holds that $f\left(I_{h}\left[u^{0}\right]\right)-\pi^{2} I_{h}\left[u^{0}\right] \geq \frac{1}{2} f\left(I_{h}\left[u^{0}\right]\right)>0$. This implies that $I_{h}\left[u^{1}\right] \geq s_{0}$, whence $f\left(I_{h}\left[u^{1}\right]\right)-\pi^{2} I_{h}\left[u^{1}\right] \geq \frac{1}{2} f\left(I_{h}\left[u^{1}\right]\right)>0$. By induction, we see that

$$
\frac{I_{h}\left[u^{n+1}\right]-I_{h}\left[u^{n}\right]}{\Delta t_{n}} \geq \frac{1}{2} f\left(I_{h}\left[u^{n}\right]\right)
$$

for all $n$.

Corollary 3.1. If $I_{h}\left[u^{0}\right] \geq s_{0}$, then $\lim _{n \rightarrow \infty}\left\|u^{n}\right\|_{p}=\infty$. 
Proof. $I_{h}\left[u^{n}\right]$ is monotone increasing in $n$. If it tends to infinity, so does $\left\|u^{n}\right\|_{p}$ by virtue of (25). If it tends to a finite limit, (since it is easily excluded that $\Delta t_{n}=\tau$ for all $n$ ) we have

$$
I_{h}\left[u^{n+1}\right]-I_{h}\left[u^{n}\right] \geq \frac{\tau f\left(I_{h}\left[u^{n}\right]\right)}{2 g\left(\left\|u^{n}\right\|_{p}\right)}
$$

whence $1 / g\left(\left\|u^{n}\right\|_{p}\right) \rightarrow 0$, which can be true if and only if $\left\|u^{n}\right\|_{p} \rightarrow \infty$.

LEMMA 3.3. Under the same assumption as in the previous lemma, we have for all sufficiently large $n$

$$
\frac{\left\|u^{n+1}\right\|_{p}-\left\|u^{n}\right\|_{p}}{\Delta t_{n}} \geq\left(-\frac{2}{h^{2}}-f(0)\right)\left\|u^{n}\right\|_{p}+f\left(\left\|u^{n}\right\|_{p}\right) .
$$

Proof. We first consider the case of $p=\infty$. In (16), let $i$ be such that $u_{i}^{n}=\max _{1 \leq j \leq N-1} u_{j}^{n}$. Then, by the positivity of $u_{j}^{n}$, we have

$$
\begin{aligned}
\left\|u^{n+1}\right\|_{\infty}=u_{i}^{n+1} & \geq\left(1-\frac{2 \Delta t_{n}}{h^{2}}\right) u_{i}^{n}+\Delta t_{n} f\left(u_{i}^{n}\right) \\
& =\left(1-\frac{2 \Delta t_{n}}{h^{2}}\right)\left\|u^{n}\right\|_{\infty}+\Delta t_{n} f\left(\left\|u^{n}\right\|_{\infty}\right),
\end{aligned}
$$

which claims more than (27).

Suppose next that $1 \leq p<\infty$. Multiply (16) by $h\left(u_{j}^{n}\right)^{p-1}$ and take the summation with respect to $j$. Since $u_{j}^{n}$ are positive, we have

$$
h \sum_{j=1}^{N-1} u_{j}^{n+1}\left(u_{j}^{n}\right)^{p-1}-h \sum_{j=1}^{N-1}\left(u_{j}^{n}\right)^{p} \geq-\frac{2 \Delta t_{n} h}{h^{2}} \sum_{j=1}^{N-1}\left(u_{j}^{n}\right)^{p}+\Delta t_{n} h \sum_{j=1}^{N-1} f\left(u_{j}^{n}\right)\left(u_{j}^{n}\right)^{p-1} .
$$

By Hölder's inequality and the positivity of $f$, this can be written as

$$
\left\|u^{n+1}\right\|_{p}\left\|u^{n}\right\|_{p}^{p-1} \geq\left(1-2 \lambda_{n}\right)\left\|u^{n}\right\|_{p}^{p}+\Delta t_{n} h \sum_{j=1}^{N-1}\left(f\left(u_{j}^{n}\right)-f(0)\right)\left(u_{j}^{n}\right)^{p-1}
$$

where we have set $\lambda_{n}=\Delta t_{n} / h^{2}$. The last term of the right hand side is estimated as follows. Let $\psi$ be defined by $\psi(x)=\left(f\left(x^{1 / p}\right)-f(0)\right) x^{(p-1) / p}$. We can verify in an elementary way that $\psi$ is convex. In fact,

$$
\psi^{\prime \prime}(x)=\frac{1}{p^{2}} f^{\prime \prime}\left(x^{1 / p}\right)+\frac{p-1}{p^{2}} x^{-1 / p-1}\left\{x^{1 / p} f^{\prime}\left(x^{1 / p}\right)-f\left(x^{1 / p}\right)+f(0)\right\} .
$$

$f^{\prime \prime}$ is nonnegative because of the convexity. Also,

$$
f(u)=f(0)+\int_{0}^{u} f^{\prime}(t) \mathrm{d} t \leq f(0)+u f^{\prime}(u) .
$$


Therefore $\psi^{\prime \prime} \geq 0$. Now, Jensen's inequality gives us

$$
\frac{1}{N} \sum_{k=1}^{N} \psi\left(\left(u_{k}^{n}\right)^{p}\right) \geq \psi\left(\frac{1}{N} \sum_{k=1}^{N}\left(u_{k}^{n}\right)^{p}\right)=\psi\left(\left\|u^{n}\right\|_{p}^{p}\right)=\left[f\left(\left\|u^{n}\right\|_{p}\right)-f(0)\right]\left\|u^{n}\right\|_{p}^{p-1} .
$$

Summing up, we obtain

$$
\left\|u^{n+1}\right\|_{p}\left\|u^{n}\right\|_{p}^{p-1} \geq\left(1-2 \lambda_{n}\right)\left\|u^{n}\right\|_{p}^{p}+\Delta t_{n}\left(f\left(\left\|u^{n}\right\|_{p}\right)-f(0)\right)\left\|u^{n}\right\|_{p}^{p-1},
$$

from which follows

$$
\left\|u^{n+1}\right\|_{p}-\left\|u^{n}\right\|_{p} \geq-\frac{2 \Delta t_{n}}{h^{2}}\left\|u^{n}\right\|_{p}+\Delta t_{n} f\left(\left\|u^{n}\right\|_{p}\right)-\Delta t_{n} f(0) .
$$

Since $\left\|u^{n}\right\|_{p}$ tends to infinity, it eventually becomes greater than one. Hence we have (27).

We now apply Lemma 3.1 with $b=1 / 2$ and $K=2 h^{-2}+f(0)$. Then, there exists a constant $s_{0}=s_{0}(h)$ such that if $\left\|u^{n_{1}}\right\|_{p} \geq s_{0}(h)$ it holds for all $n \geq n_{1}$ that

$$
\frac{\left\|u^{n+1}\right\|_{p}-\left\|u^{n}\right\|_{p}}{\Delta t_{n}} \geq \frac{1}{2} f\left(\left\|u^{n}\right\|_{p}\right) .
$$

Because of 3.1, such an $n_{1}$ exists.

Proof of Theorem 3.2. We set

$$
T_{*}=\liminf _{h \rightarrow 0} T(\tau, h) \quad \text { and } \quad T^{*}=\limsup _{h \rightarrow 0} T(\tau, h),
$$

both of which may be infinite at this stage. We will prove the convergence by showing $T_{*} \geq T$ and $T^{*} \leq T$.

First, we assume that $T_{*}<T$ and derive a contradiction. This part of the proof is actually the same as in [12]. If $T_{*}<T$, then there exists an $h$ and a $\tau$ such that $\tau / h^{2} \leq 1 / 2, h$ is smaller than any prescribed positive number, and

$$
T(\tau, h) \leq \frac{T+T_{*}}{2} .
$$

Let $\left\{u_{k}^{n}(\tau, h)\right\}$ denote the solution corresponding to the parameter $(\tau, h)$. Accordingly we have $t_{n}(\tau, h) \leq\left(T+T_{*}\right) / 2<T$ for all $n$. On the other hand, by Corollary 3.1, we have $\left\|u_{k}^{n}(\tau, h)\right\|_{p} \rightarrow \infty$ as $n \rightarrow \infty$, which contradicts Theorem 3.1 , since the theorem guarantees that $\left\{u_{k}^{n}(\tau, h)\right\}$ converge to $u$ on $\left[0,\left(T+T_{*}\right) / 2\right]$, where $u$ is smooth. We therefore have $T \leq T_{*}$.

Proof of $T^{*} \leq T$. Let us assume that $T^{*}>T$. Take a $(\tau, h)$ such that $\tau / h^{2} \leq$ $1 / 2, h$ is smaller than any prescribed positive number, and

$$
T(\tau, h)>\frac{T+T^{*}}{2} .
$$


Now, let an arbitrary $M>0$ is given. Then Theorem 3.1 guarantees, by choosing smaller $h$ and $\tau$ are chosen if necessary, the existence of an $\tilde{n}$ such that

$$
t_{\tilde{n}}<T, \quad\left\|u^{n}(\tau, h)\right\|_{p} \geq M \quad(\tilde{n} \leq \forall n) .
$$

We may assume without losing generality that $g\left(\left\|u^{n}\right\|_{p}\right) \geq 1$ for all $n \geq \tilde{n}$. We then consider the following finite difference equation

$$
\frac{v^{n+1}-v^{n}}{\Delta \tau_{n}}=\frac{\tau f\left(v^{n}\right)}{2 g\left(v^{n}\right)}, \quad(\tilde{n} \leq n) ; \quad v^{\tilde{n}}=\left\|u^{\tilde{n}}\right\|_{p} .
$$

Now, by Lemma 3.3 we have for all $n \geq \tilde{n}$

$$
\left\|u^{n+1}(\tau, h)\right\|_{p} \geq\left\|u^{n}(\tau, h)\right\|_{p}+\frac{1}{2} \frac{\tau}{g\left(\left\|u^{n}\right\|_{p}\right)} f\left(\left\|u^{n}(\tau, h)\right\|_{p}\right) .
$$

It can be easily checked by the monotonicity (assumption (iv) of the present theorem) that $v^{n} \leq\left\|u^{n}\right\|_{p}$ for all $n \geq \tilde{n}$.

We now have

$$
\begin{aligned}
T(\tau, h) & =t_{\tilde{n}}+\sum_{k=0}^{\infty} \frac{\tau}{g\left(\left\|u^{\tilde{n}+k}\right\|_{p}\right)} \\
& \leq t_{\tilde{n}}+\sum_{k=0}^{\infty} \frac{\tau}{g\left(v^{\tilde{n}+k}\right)} \\
& <T+2 \int_{M}^{\infty} \frac{d s}{f(s)}+\tau \int_{M}^{\infty} \frac{f^{\prime}(s)}{f(s) g(s)} d s .
\end{aligned}
$$

Here use has been made of Corollary 2.1. Since $M$ can be arbitrarily large, we may assume that

$$
2 \int_{M}^{\infty} \frac{d s}{f(s)}+\tau \int_{M}^{\infty} \frac{f^{\prime}(s)}{f(s) g(s)} d s<\frac{T^{*}-T}{2}
$$

Thus, (31) implies that $T(\tau, h)<\left(T+T^{*}\right) / 2$, which contradicts $(28)$.

Since we have proved $T \leq T_{*} \leq T^{*} \leq T$, we are done.

We now consider a special case that $f(s)=s^{1+\alpha}$ and $g(s)=s^{\gamma} \cdot L^{2}$-norm is used. In [12], Nakagawa chose $\gamma=1$ (when $\alpha=1$ ) and Chen chose $\gamma=\alpha$ in [6]. Let us see whether their choices were the best choice or not.

Corollary 2.2 shows that the upper bound of the error decreases as $\gamma$ increases. Therefore one might be tempted to conclude that a larger $\gamma$ is better. This view may be strengthened by the following fact, which can be proved without difficulty: the numerical blow-up time becomes infinite if uniform time-mesh is used. Namely $T(\tau, h)=\infty$, hence the error is infinite, if $\gamma=0$. However, we must be particularly careful in this issue. In fact, the larger $\gamma$ becomes, the smaller the time-mesh size becomes. Consequently, the computation becomes unnecessarily slow. Let us show 
an experiment: In it, we put $\alpha=1$ and $u(0, x)=100 \sin (\pi x)$ and set a large threshold $U_{0}=10^{6}$, and we stop the computation when $\left\|u^{n}\right\|_{2}>U_{0}$. The $n$ where we stopped is denoted by $n(\infty)$. Then our experiments gave Table 1 . The number of steps needed to achieve $\left\|u^{n}\right\|_{2}>U_{0}$ remarkably increases with $\gamma$. Therefore the best $\gamma$ may be determined by the balance of error and cost. We have so far no evidence that $\gamma=\alpha$ is the best, nor do we have anything which suggests that $\gamma=\alpha$ is not the best. In fact, how to measure the suitability of $\gamma$ is itself a problem.

Table 1. $\gamma$ vs. $T(\tau)$ and $n(\infty)$ with initial data $u_{0}(x)=100 \sin (\pi x) . \quad \alpha=1, p=2$, $\lambda=0.3, h=0.01$

\begin{tabular}{|c|c|l|}
\hline$\gamma$ & \multicolumn{1}{|c|}{$T(\tau)$} & \multicolumn{1}{|c|}{$n(\infty)$} \\
\hline 0.5 & 0.010992052 & 5228 \\
\hline 0.6 & 0.010988277 & 9255 \\
\hline 0.7 & 0.010986278 & 16752 \\
\hline 0.8 & 0.010985189 & 31190 \\
\hline 0.9 & 0.010984581 & 60193 \\
\hline 1.0 & 0.010984235 & 121563 \\
\hline 1.1 & 0.010984035 & 259646 \\
\hline 1.2 & 0.010983918 & 592340 \\
\hline 1.3 & 0.010983849 & 1452938 \\
\hline 1.4 & 0.010983808 & 3836344 \\
\hline 1.5 & 0.010983783 & 10852257 \\
\hline
\end{tabular}

Finally, we consider the dependence of $T(\tau, h)$ on $h$ and $\tau$. In the case of ODE, Theorem 2.1 tells us that $T(\tau)$ converges to $T$ from above as $\tau \downarrow 0$. Actually, our numerical experiments shows that $T(\tau)$ decreases monotonically as $\tau \downarrow 0$. In the case of PDE, however, our numerical experiments (Table 2) seem to suggest that $T(\tau, h) \uparrow T$ as $h \downarrow 0$. We do not know the reason.

Table 2. $h$ vs. $T(\tau)$ with some initial data. $p(x)=50 \sin (3 \pi x)+200 x(1-x)$

\begin{tabular}{|c|c|c|c|}
\hline & \multicolumn{3}{|c|}{$T(\tau)$} \\
\hline$N=1 / h$ & $u_{0}(x)=100 \sin (\pi x)$ & $u_{0}(x)=400 x(1-x)$ & $u_{0}(x)=p(x)$ \\
\hline 16 & 0.010975079567 & 0.010821160594 & 0.023026708637 \\
\hline 32 & 0.010981629673 & 0.010823094329 & 0.023287362291 \\
\hline 64 & 0.010983747246 & 0.010824043459 & 0.023342085859 \\
\hline 128 & 0.010984380128 & 0.010824380585 & 0.023357220435 \\
\hline 256 & 0.010984567576 & 0.010824492970 & 0.023360793794 \\
\hline 512 & 0.010984618838 & 0.010824524941 & 0.023361750438 \\
\hline 1024 & 0.010984628834 & 0.010824531271 & 0.023361987222 \\
\hline
\end{tabular}

REMARK 3.3. In the present paper we focus our attention to the Dirichlet boundary condition. However, we can handle the Neumann boundary condition $u_{x}(t, 0)=u_{x}(t, 1)=0$ in quite the same way, if we follow the proof of Chen $[6]$. 


\section{Asymptotic behavior of the numerical solution when $f(u)=u^{1+\alpha}$}

In this section, we consider the equation (13) with the nonlinear term $f(u)=$ $u^{1+\alpha}$. The difference scheme (16) is accordingly written as

$$
\frac{u_{j}^{n+1}-u_{j}^{n}}{\Delta t_{n}}=\frac{u_{j+1}^{n}-2 u_{j}^{n}+u_{j-1}^{n}}{h^{2}}+\left(u_{j}^{n}\right)^{1+\alpha} .
$$

We consider this with the Dirichlet boundary condition (17). We define

$$
\Delta t_{n}=\tau \min \left\{1, \frac{1}{\left\|u^{n}\right\|_{p}^{\alpha}}\right\}
$$

where $1 \leq p \leq \infty$ is a fixed parameter.

From now on until the end of the present paper, we assume that the initial data satisfies the following conditions:

(A1) $u_{0}(x)$ is spatially symmetric about the point $x=1 / 2$.

(A2) $u_{0}(x)$ is monotone increasing in $[0,1 / 2]$.

We always assume that $u_{0}(x) \geq 0$ everywhere.

REMARK 4.1. Under the assumptions (A1) and (A2), once a solution blows up, the single-point blow-up occurs to the solution of (13) (14) (15). Specifically we have

$$
\lim _{t \rightarrow T} u\left(t, \frac{1}{2}\right)=\infty, \quad \text { and } \quad \limsup _{t \rightarrow T} u(t, x)<\infty \quad \text { for } \quad x \neq \frac{1}{2} .
$$

For proof, see [8], [17].

Henceforth we assume that $N$ is even and set $N=2 m$, so that $x_{m}=1 / 2$.

REMARK 4.2. Let $\left\{u_{j}^{n}\right\}$ be a solution of (32) with (17). If $u_{0}(x)$ satisfies (A1) and (A2), then

(i) $u_{m-j}^{n}=u_{m+j}^{n}(j=1, \ldots, m, n=0,1, \ldots)$;

(ii) $0<u_{j}^{n}<u_{j+1}^{n}(j=1, \ldots, m-1, n \geq m-1)$.

These facts will be used later.

Since $u_{m}^{n}$ is the largest among $\left\{u_{j}^{n}\right\}$, we have $\lim _{n \rightarrow \infty} u_{m}^{n}=+\infty$ if $\left\{u_{j}^{n}\right\}$ blows up.

THEOREM 4.1. Let $\left\{u_{j}^{n}\right\}$ be a solution of (32) and (17) and suppose that the initial data is large enough for $u_{m}^{n}$ to blow up. Assume that (A1) and (A2) hold. Then $u_{m-1}^{n}$ is bounded by a constant depending only on $u_{0}$ and $h$ if $\alpha>1$, while $\lim _{n \rightarrow \infty} u_{m-1}^{n}=+\infty$ if $\alpha \leq 1$. Moreover, $u_{m-2}^{n}$ remains bounded by a constant depending only on $u_{0}$ and $h$ if $\alpha=1$.

This theorem was discovered and proved by Chen [6] in the case of the Neumann boundary condition and $p=1$, and in the case of the Dirichlet boundary condition and $p=2$. We are now going to generalize his theorem in the following way. 
THEOREM 4.2. Under the assumptions of Theorem 4.1. Let $k$ be any positive integer and assume that

$$
\frac{1}{k+1}<\alpha \leq \frac{1}{k}
$$

Then the solution blows up exactly at the central $2 k+1$ points and it is bounded at all the other points. Namely,

$$
\lim _{n \rightarrow \infty} u_{j}^{n}=\infty \quad \text { if and only if }|j-m| \leq k .
$$

In order to prove this theorem, we use two sequences $\left\{a_{n}\right\}$ and $\left\{b_{n}\right\}$ defined as follows:

$$
a_{n}=\frac{u_{m-1}^{n}}{u_{m}^{n}}, \quad b_{n}=\frac{\left(u_{m}^{n}\right)^{\alpha}}{\left\|u^{n}\right\|_{p}^{\alpha}} .
$$

We begin our analysis with the following lemmas.

LEMma 4.1. If $0<\alpha \leq 1$, then $\lim _{n \rightarrow \infty} u_{m-1}^{n}=\lim _{n \rightarrow \infty} u_{m+1}^{n}=\infty$.

Lemma 4.2. For any $\alpha>0$, we have

$$
\lim _{n \rightarrow \infty} a_{n}=0
$$

and

$$
\lim _{n \rightarrow \infty} b_{n}=h^{-\alpha / p} .
$$

These two lemmas were proved in Chen [6] in the case of the Neumann boundary condition and $p=1$. In fact, the proof in Chen [6] is valid in our general case, too. We may therefore omit the proof. In what follows, we set $\lambda_{n}=\Delta t_{n} / h^{2}$ for notational convenience. (Recall that $\lambda=\tau / h^{2}$.)

Corollary 4.1. For any $\alpha>0$, we have

$$
\lim _{n \rightarrow \infty} \frac{u_{m}^{n}}{u_{m}^{n+1}}=\frac{1}{1+\tau h^{-\alpha / p}}
$$

Proof. We have by (32)

$$
u_{m}^{n+1}=2 \lambda_{n} u_{m-1}^{n}+\left(1-2 \lambda_{n}+\tau b_{n}\right) u_{m}^{n} .
$$

The present corollary can be easily derived from (36) by letting $n \rightarrow \infty$.

Lemma 4.3. For any $\alpha>0$, we have

$$
\lim _{n \rightarrow \infty} \frac{u_{m-1}^{n+1}-\left\{1-2 \lambda_{n}+\Delta t_{n}\left(u_{m-1}^{n}\right)^{\alpha}\right\} u_{m-1}^{n}}{u_{m-1}^{n+2}-\left\{1-2 \lambda_{n+1}+\Delta t_{n+1}\left(u_{m-1}^{n+1}\right)^{\alpha}\right\} u_{m-1}^{n+1}}=\left(1+\tau h^{-\alpha / p}\right)^{\alpha-1} .
$$


Proof. By (34) and (35), we have

$$
\lim _{n \rightarrow \infty} \frac{\lambda_{n}}{\lambda_{n+1}}=\lim _{n \rightarrow \infty} \frac{\left\|u^{n+1}\right\|_{p}^{\alpha}}{\left\|u^{n}\right\|_{p}^{\alpha}}=\lim _{n \rightarrow \infty}\left(\frac{u_{m}^{n+1}}{u_{m}^{n}}\right)^{\alpha}=\left(1+\tau h^{-\alpha / p}\right)^{\alpha} .
$$

This relation, (33), and (35) yield

$$
\begin{aligned}
& \frac{u_{m-1}^{n+1}-\left\{1-2 \lambda_{n}+\Delta t_{n}\left(u_{m-1}^{n}\right)^{\alpha}\right\} u_{m-1}^{n}}{u_{m-1}^{n+2}-\left\{1-2 \lambda_{n+1}+\Delta t_{n+1}\left(u_{m-1}^{n+1}\right)^{\alpha}\right\} u_{m-1}^{n+1}} \\
& =\frac{\lambda_{n}\left(u_{m}^{n}+u_{m-2}^{n}\right)}{\lambda_{n+1}\left(u_{m}^{n+1}+u_{m-2}^{n+1}\right)} \\
& =\frac{\lambda_{n}}{\lambda_{n+1}} \cdot\left(\frac{u_{m}^{n}}{u_{m}^{n+1}}+\frac{u_{m-2}^{n}}{u_{m}^{n+1}}\right) /\left(1+\frac{u_{m-2}^{n+1}}{u_{m}^{n+1}}\right) \\
& \rightarrow\left(1+\tau h^{-\alpha / p}\right)^{\alpha} \cdot \frac{1}{1+\tau h^{-\alpha / p}}=\left(1+\tau h^{-\alpha / p}\right)^{\alpha-1} .
\end{aligned}
$$

LEMMA 4.4. For all $\alpha>0, \lim _{n \rightarrow \infty} u_{m-1}^{n} / u_{m-1}^{n+1}$ exists and is equal to $(1+$ $\left.\tau h^{-\alpha / p}\right)^{\min \{1, \alpha\}-1}$.

Proof. We use (32) with $j=m-1$ :

$$
u_{m-1}^{n+1}=\lambda_{n} u_{m}^{n}+\lambda_{n} u_{m-2}^{n}+\left(1-2 \lambda_{n}+\Delta t_{n}\left(u_{m-1}^{n}\right)^{\alpha}\right) u_{m-1}^{n},
$$

which is written as

$$
1=\lambda_{n} \frac{u_{m}^{n}}{u_{m-1}^{n+1}}+\lambda_{n} \frac{u_{m-2}^{n}}{u_{m-1}^{n+1}}+\left(1-2 \lambda_{n}+\tau b_{n} a_{n}^{\alpha}\right) \frac{u_{m-1}^{n}}{u_{m-1}^{n+1}} .
$$

Since $u_{m}^{n}$ tends to infinity, it holds that

$$
\frac{\lambda_{n} u_{m}^{n}}{u_{m-1}^{n+1}}=\frac{\lambda b_{n}\left(u_{m}^{n}\right)^{1-\alpha}}{u_{m-1}^{n+1}} \rightarrow 0 \quad \text { as } \quad n \rightarrow \infty
$$

if $\alpha>1$. If $\alpha=1$, then $u_{m-1}^{n}$, too, tends to infinity and we have the same result. By letting $n \rightarrow \infty$ in (40), the present lemma in the case of $\alpha \geq 1$ follows.

We now assume that $\alpha<1$. In this case, (40) is of no help, and we use Lemma 4.3 instead. For notational convenience, we set $w_{n}=u_{m-1}^{n} / u_{m-1}^{n+1}$. We note that, for sufficiently large $n$, we have

$$
0<w_{n}<1
$$

In fact, we have by (39) $u_{m-1}^{n+1}-u_{m-1}^{n} \geq \lambda_{n}\left(u_{m}^{n}-2 u_{m-1}^{n}\right)$, the right hand side of which is positive for sufficiently large $n$ 's by virtue of (33). We now write (37) as

$$
\lim _{n \rightarrow \infty} w_{n+1} \frac{1-\left(1+\gamma_{n}\right) w_{n}}{1-\left(1+\gamma_{n+1}\right) w_{n+1}}=\left(1+\tau h^{-\alpha / p}\right)^{\alpha-1},
$$


where $\gamma_{n}=-2 \lambda_{n}+\Delta t_{n}\left(u_{m-1}^{n}\right)^{\alpha}=-2 \lambda_{n}+\tau b_{n} a_{n}^{\alpha}$ satisfies $\lim _{n \rightarrow \infty} \gamma_{n}=0$. The conclusion of the present lemma follows easily from this, once we have shown that $\lim _{n \rightarrow \infty} w_{n}$ exists and is less than one.

We follow Chen's recipe, and assume that

$$
\liminf _{n \rightarrow \infty} w_{n}<\limsup _{n \rightarrow \infty} w_{n}
$$

Let $r$ be any number such that $\liminf _{n \rightarrow \infty} w_{n}<r<\limsup _{n \rightarrow \infty} w_{n}$. Then there exists a subsequence $\left\{n_{k}\right\}$ such that

$$
w_{n_{k}} \leq r \quad \text { and } \quad r<w_{n_{k}+1}
$$

This implies that

$u_{m-1}^{n_{k}+1}-\left\{1-2 \lambda_{n_{k}}+\Delta t_{n_{k}}\left(u_{m-1}^{n_{k}}\right)^{\alpha}\right\} u_{m-1}^{n_{k}} \geq\left\{1-r+2 r \lambda_{n_{k}}-r \Delta t_{n_{k}}\left(u_{m-1}^{n_{k}}\right)^{\alpha}\right\} u_{m-1}^{n_{k}+1}$

and

$$
\begin{aligned}
& u_{m-1}^{n_{k}+2}-\left\{1-2 \lambda_{n_{k}+1}+\Delta t_{n_{k}+1}\left(u_{m-1}^{n_{k}+1}\right)^{\alpha}\right\} u_{m-1}^{n_{k}+1} \\
& <\left(1-r+2 r \lambda_{n_{k}+1}\right) u_{m-1}^{n_{k}+2}-\Delta t_{n_{k}+1}\left(u_{m-1}^{n_{k}+1}\right)^{1+\alpha} \\
& <\left(1-r+2 r \lambda_{n_{k}+1}\right) u_{m-1}^{n_{k}+2} .
\end{aligned}
$$

These two inequalities give us

$$
\begin{aligned}
& \frac{u_{m-1}^{n_{k}+1}-\left\{1-2 \lambda_{n_{k}}+\Delta t_{n_{k}}\left(u_{m-1}^{n_{k}}\right)^{\alpha}\right\} u_{m-1}^{n_{k}}}{u_{m-1}^{n_{k}+2}-\left\{1-2 \lambda_{n_{k}+1}+\Delta t_{n_{k}+1}\left(u_{m-1}^{n_{k}+1}\right)^{\alpha}\right\} u_{m-1}^{n_{k}+1}} \\
& >\frac{\left\{1-r+2 r \lambda_{n_{k}}-r \Delta t_{n_{k}}\left(u_{m-1}^{n_{k}}\right)^{\alpha}\right\} u_{m-1}^{n_{k}+1}}{\left(1-r+2 r \lambda_{n_{k}+1}\right) u_{m-1}^{n_{k}+2}}
\end{aligned}
$$

Note that $\Delta t_{n_{k}}\left(u_{m-1}^{n_{k}}\right)^{\alpha}=\tau b_{n_{k}} a_{n_{k}}^{\alpha} \rightarrow 0$ as $k \rightarrow \infty$, which together with Lemma 4.3 implies that

$$
\begin{aligned}
\left(1+\tau h^{-\alpha / p}\right)^{\alpha-1} & \geq \limsup _{k \rightarrow \infty} \frac{\left\{1-r+2 r \lambda_{n_{k}}-\Delta t_{n_{k}}\left(u_{m-1}^{n_{k}}\right)^{\alpha}\right\} u_{m-1}^{n_{k}+1}}{\left(1-r+2 r \lambda_{n_{k}+1}\right) u_{m-1}^{n_{k}+2}} \\
& =\limsup _{k \rightarrow \infty} w_{n_{k}+1} \geq r .
\end{aligned}
$$

On the other hand, we can also find a subsequence $\left\{\bar{n}_{j}\right\}$ such that

$$
w_{\bar{n}_{j}}>r \quad \text { and } \quad w_{\bar{n}_{j}+1} \leq r .
$$

In exactly the same way, we obtain $r \leq\left(1+\tau h^{-\alpha / p}\right)^{\alpha-1}$. We therefore have $r=\left(1+\tau h^{-\alpha / p}\right)^{\alpha-1}$. But this is a contradiction because $r$ was chosen arbitrarily between $\liminf \operatorname{in}_{n \rightarrow \infty} w_{n}$ and $\limsup _{n \rightarrow \infty} w_{n}$. Hence, $\lim _{n \rightarrow \infty} w_{n}$ exists and $0 \leq$ $\lim _{n \rightarrow \infty} w_{n} \leq 1$. 
Let $A=\lim _{n \rightarrow \infty} w_{n}$. We next prove that $A<1$. Assume that $A=1$. Then (41) shows that

$$
\lim _{n \rightarrow \infty} \frac{\xi_{n}}{\xi_{n+1}}=\left(1+\tau h^{-\alpha / p}\right)^{\alpha-1}<1,
$$

where $\xi_{n}=1-\left(1+\gamma_{n}\right) w_{n}$. We may take a $\rho<1$ such that for all sufficiently large $n$ it holds that $\xi_{n} / \xi_{n+1}<\rho$, i.e., $\rho^{-1} \xi_{n}<\xi_{n+1}$. On the other hand, since $A=1$, the sequence $\xi_{n}$ tends to zero. This clearly contradicts, and the proof is completed.

Corollary 4.2. For all $\alpha>0$, we have

$$
\lim _{n \rightarrow \infty} \frac{a_{n+1}}{a_{n}}=\left(1+\tau h^{-\alpha / p}\right)^{-\min \{1, \alpha\}} .
$$

This is a direct consequence of (35) and Lemma 4.4.

REMARK 4.3. We can write (39) as

$$
\frac{u_{m-1}^{n+1}}{u_{m-1}^{n}}=\lambda b_{n} \frac{\left(u_{m}^{n}\right)^{1-\alpha}}{u_{m-1}^{n}}+\lambda_{n} \frac{u_{m-2}^{n}}{u_{m-1}^{n}}+1-2 \lambda_{n}+\tau b_{n} a_{n}^{\alpha} .
$$

Then Lemma 4.4 implies that, for $\alpha<1$,

$$
\frac{\left(u_{m}^{n}\right)^{1-\alpha}}{u_{m-1}^{n}} \rightarrow \frac{\left(1+\tau h^{-\alpha / p}\right)^{1-\alpha}-1}{\lambda h^{-\alpha / p}} \in(0, \infty) .
$$

This will be used later.

Lemma 4.5. For $\alpha \leq 1 / 2$, we have $\lim _{n \rightarrow \infty} u_{m-2}^{n}=\infty$.

Proof. We have by (32)

$$
\begin{aligned}
u_{m-2}^{n+1} & =\lambda_{n}\left(u_{m-1}^{n}+u_{m-3}^{n}\right)+\left\{1-2 \lambda_{n}+\Delta t_{n}\left(u_{m-2}^{n}\right)^{\alpha}\right\} u_{m-2}^{n} \\
& \geq \lambda_{n} u_{m-1}^{n}+\left(1-2 \lambda_{n}\right) u_{m-2}^{n} .
\end{aligned}
$$

The first term of the right hand side is written as follows:

$$
\lambda_{n} u_{m-1}^{n}=\lambda b_{n}\left\{\frac{u_{m-1}^{n}}{\left(u_{m}^{n}\right)^{1-\alpha}}\right\}^{\alpha /(1-\alpha)}\left(u_{m-1}^{n}\right)^{(1-2 \alpha) /(1-\alpha)} .
$$

This and (43) prove that $0<\liminf _{n \rightarrow \infty} \lambda_{n} u_{m-1}^{n}=M \leq \infty$ if $\alpha \leq 1 / 2$. Then (44) gives us

$$
\liminf _{n \rightarrow \infty} u_{m-2}^{n+1} \geq M+\liminf _{n \rightarrow \infty} u_{m-2}^{n},
$$

which implies that $\lim _{n \rightarrow \infty} u_{m-2}^{n}=\infty$.

Lemma 4.6. For $1 / 2<\alpha \leq 1,\left\{u_{m-2}^{n}\right\}_{n=1}^{\infty}$ remains bounded. 
To prove this, we need the following lemma, which was used by Chen [6].

LEMMA 4.7. Suppose that a positive sequence $\left\{x_{n}\right\}$ satisfies

$$
x_{n+1} \leq A_{n} x_{n}+B_{n}, \quad n=1,2, \ldots
$$

where the coefficients $A_{n}$ and $B_{n}$ are positive and satisfy

$$
\prod_{n=1}^{\infty} A_{n}<\infty, \quad \sum_{n=1}^{\infty} B_{n}<\infty
$$

Then the sequence $\left\{x_{n}\right\}$ is bounded.

We omit the proof since it is elementary. we have

Proof of Lemma 4.6. From the difference scheme (32) and $u_{m-3}^{n} \leq u_{m-2}^{n}$,

$$
\begin{aligned}
u_{m-2}^{n+1} & =\lambda_{n} u_{m-1}^{n}+\left(1-2 \lambda_{n}\right) u_{m-2}^{n}+\lambda_{n} u_{m-3}^{n}+\Delta t_{n}\left(u_{m-2}^{n}\right)^{\alpha+1} \\
& \leq \lambda_{n} u_{m-1}^{n}+\left\{1+\Delta t_{n}\left(u_{m-2}^{n}\right)^{\alpha}\right\} u_{m-2}^{n} \\
& \leq \lambda_{n} u_{m-1}^{n}+\left(1+\tau b_{n} a_{n}^{\alpha}\right) u_{m-2}^{n} \\
& \leq \lambda_{n} u_{m-1}^{n}+\left(1+\tau h^{-\alpha / p} a_{n}^{\alpha}\right) u_{m-2}^{n} \equiv B_{n}+A_{n} u_{m-2}^{n} .
\end{aligned}
$$

By Corollary 4.2, we have $\sum_{n=0}^{\infty} a_{n}^{\alpha}<\infty$. Consequently,

$$
\prod_{n=0}^{\infty} A_{n} \leq \prod_{n=0}^{\infty} \exp \left(\tau h^{-\alpha / p} a_{n}^{\alpha}\right)=\exp \left(\tau h^{-\alpha / p} \sum_{n=0}^{\infty} a_{n}^{\alpha}\right)<\infty .
$$

The inequality $\sum_{n=1}^{\infty} B_{n}<\infty$ is proved by Corollary 4.1 and Lemma 4.4 as follows:

$$
\begin{aligned}
\lim _{n \rightarrow \infty} \frac{B_{n+1}}{B_{n}} & =\lim _{n \rightarrow \infty} \frac{\left(u_{m}^{n}\right)^{\alpha}}{\left(u_{m}^{n+1}\right)^{\alpha}} \frac{u_{m-1}^{n+1}}{u_{m-1}^{n}}=\left(1+\tau h^{-\alpha / p}\right)^{-\alpha}\left(1+\tau h^{-\alpha / p}\right)^{1-\alpha} \\
& =\left(1+\tau h^{-\alpha / p}\right)^{1-2 \alpha}<1,
\end{aligned}
$$

for $1 / 2<\alpha \leq 1$. Thus, the desired result follows from Lemma 4.7.

By the preceding two lemmas, we have shown that there are exactly three blowup points if $1 / 2<\alpha \leq 1$ while there are at least five blow-up points for $\alpha \leq 1 / 2$. We naturally wonder about the exact number of blow-up points for $\alpha \leq 1 / 2$. To study this, it is necessary to know the relationship of $u_{m-1}^{n}$ and $u_{m-2}^{n}$ when $\alpha \leq 1 / 2$.

We define $c_{n}$ by $c_{n}=u_{m-2}^{n} / u_{m-1}^{n}$ for $n=0,1,2, \ldots$ and prove the following:

LEMMA 4.8. For $\alpha<1$, we have $\lim _{n \rightarrow \infty} c_{n}=0$.

Proof. The inequality (45) implies that

$$
c_{n+1} \leq \lambda_{n} \frac{u_{m-1}^{n}}{u_{m-1}^{n+1}}+\left\{1+\Delta t_{n}\left(u_{m-2}^{n}\right)^{\alpha}\right\} \frac{u_{m-1}^{n}}{u_{m-1}^{n+1}} c_{n} .
$$


Then, by Lemma 4.4,

$$
\limsup _{n \rightarrow \infty} c_{n+1} \leq 0+\left(1+\tau h^{-\alpha / p}\right)^{\alpha-1} \limsup _{n \rightarrow \infty} c_{n},
$$

which implies that $\limsup _{n \rightarrow \infty} c_{n}=0$, since $\alpha<1$.

With the help of this lemma, we can prove the following lemma in exactly the same way as before.

Lemma 4.9. If $\alpha \leq 1 / 3$, we have $\lim _{n \rightarrow \infty} u_{m-3}^{n}=\infty$. If $1 / 3<\alpha \leq 1 / 2$, $\left\{u_{m-3}^{n}\right\}_{n=1}^{\infty}$ remains bounded.

The process above can be continued further, and we can prove Theorem 4.2 by induction:

Proof of Theorem 4.2. We show by induction on $k$ that, for $\alpha \leq 1 / k$, the following results [R1] and [R2] hold true:

[R1] For each $j=1,2, \ldots, k$.

$$
\begin{gathered}
\lim _{n \rightarrow \infty} u_{m-j}^{n}=\infty, \\
\lim _{n \rightarrow \infty} \frac{u_{m-j}^{n}}{u_{m-j}^{n+1}}=\left(1+\tau h^{-\alpha / p}\right)^{\min \{1, j \alpha\}-1},
\end{gathered}
$$

[R2] Except in the case that $j=k$ and $\alpha=1 / k$, it holds that

$$
\lim _{n \rightarrow \infty} \frac{\left(u_{m-j}^{n}\right)^{\frac{1-(j-1) \alpha}{1-j \alpha}}}{u_{m-j+1}^{n}} \text { exists, and } 0<\lim _{n \rightarrow \infty} \frac{\left(u_{m-j}^{n}\right)^{\frac{1-(j-1) \alpha}{1-j \alpha}}}{u_{m-j+1}^{n}}<\infty,
$$

and

$$
\lim _{n \rightarrow \infty} \frac{u_{m-j-1}^{n}}{u_{m-j}^{n}}=0 .
$$

The 'if' part of Theorem 4.2 follows from (46). Also, the 'only if' part, namely the boundedness of $u_{j}^{n}$ for $1 \leq j \leq m-k-1$ when $1 /(k+1)<\alpha$ will be proved in the course of the proof of (46)-(49). This completes the proof.

The relations (46)-(49) are valid for $k=1$ by Theorem 4.1, Lemmas 4.4, 4.8 and Remark 4.3. Assume that they hold for $k-1$. We will show that the results also hold for $k$ as far as $\alpha \leq 1 / k$. In fact, by virtue of (32), we have

$$
\begin{aligned}
u_{m-k}^{n+1} \geq & \lambda_{n} u_{m-(k-1)}^{n}+\left(1-2 \lambda_{n}\right) u_{m-k}^{n} \\
= & \lambda b_{n}\left\{\frac{\left(u_{m-1}^{n}\right)^{\frac{1}{1-\alpha}}}{u_{m}^{n}}\right\}^{\alpha} \times\left\{\frac{\left(u_{m-2}^{n}\right)^{\frac{1-\alpha}{1-2 \alpha}}}{u_{m-1}^{n}}\right\}^{\frac{\alpha}{1-\alpha}} \times \cdots \\
& \times\left\{\frac{\left(u_{m-k+1}^{n}\right)^{\frac{1-(k-2) \alpha}{1-(k-1) \alpha}}}{u_{m-k+2}^{n}}\right\}^{\frac{\alpha}{1-(k-2) \alpha}}\left(u_{m-k+1}^{n}\right)^{\frac{1-k \alpha}{1-(k-1) \alpha}}+\left(1-2 \lambda_{n}\right) u_{m-k}^{n} .
\end{aligned}
$$


We then apply (46) and (48) for $1 \leq j \leq k-1$ to obtain

$$
\liminf _{n \rightarrow \infty} u_{m-k}^{n+1} \geq M+\liminf _{n \rightarrow \infty} u_{m-k}^{n}
$$

for some $0<M \leq \infty$. This implies that $\liminf _{n \rightarrow \infty} u_{m-k}^{n}=\infty$, thus (46) holds for $k$.

To prove (47) for $j=k$, we note (see (38)) that

$$
\begin{aligned}
\frac{u_{m-k}^{n+1}-\left\{1-2 \lambda_{n}+\Delta t_{n}\left(u_{m-k}^{n}\right)^{\alpha}\right\} u_{m-k}^{n}}{u_{m-k}^{n+2}-\left\{1-2 \lambda_{n+1}+\Delta t_{n+1}\left(u_{m-k}^{n+1}\right)^{\alpha}\right\} u_{m-k}^{n+1}} & =\frac{\lambda_{n}\left(u_{m-k+1}^{n}+u_{m-k-1}^{n}\right)}{\lambda_{n+1}\left(u_{m-k+1}^{n+1}+u_{m-k-1}^{n+1}\right)} \\
& \rightarrow\left(1+\tau h^{-\alpha / p}\right)^{k \alpha-1}
\end{aligned}
$$

as $n \rightarrow \infty$, since (47) and (49) are valid for $k-1$. In exactly the same manner as in Lemma 4.4, we can show that $\lim _{n \rightarrow \infty} u_{m-k}^{n} / u_{m-k}^{n+1}$ exists and is equal to $\left(1+\tau h^{-\alpha / p}\right)^{k \alpha-1}$. Thus (47) was proved for $k$.

By (32), we have

$$
1=\lambda_{n} \frac{u_{m-k+1}^{n}}{u_{m-k}^{n+1}}+\lambda_{n} \frac{u_{m-k-1}^{n}}{u_{m-k}^{n+1}}+\left(1-2 \lambda_{n}\right) \frac{u_{m-k}^{n}}{u_{m-k}^{n+1}}+\tau b_{n} \frac{\left(u_{m-k}^{n}\right)^{\alpha}}{\left(u_{m}^{n}\right)^{\alpha} u_{m-k}^{n+1}},
$$

which shows that

$$
\lim _{n \rightarrow \infty} \lambda_{n} \frac{u_{m-k+1}^{n}}{u_{m-k}^{n+1}}=1-\left(1+\tau h^{-\alpha / p}\right)^{k \alpha-1} .
$$

Note that

$$
\begin{aligned}
\lambda_{n} \frac{u_{m-k+1}^{n}}{u_{m-k}^{n+1}}=\lambda & b_{n}\left\{\frac{\left(u_{m-1}^{n}\right)^{\frac{1}{1-\alpha}}}{u_{m}^{n}}\right\}^{\alpha} \times\left\{\frac{\left(u_{m-2}^{n}\right)^{\frac{1-\alpha}{1-2 \alpha}}}{u_{m-1}^{n}}\right\}^{\frac{\alpha}{1-\alpha}} \times \cdots \\
& \times\left\{\frac{\left(u_{m-k+1}^{n}\right)^{\frac{1-(k-2) \alpha}{1-(k-1) \alpha}}}{u_{m-k+2}^{n}}\right\}^{\frac{\alpha}{1-(k-2) \alpha}} \cdot \frac{\left(u_{m-k+1}^{n}\right)^{\frac{1-k \alpha}{1-(k-1) \alpha}}}{u_{m-k}^{n}} \cdot \frac{u_{m-k}^{n}}{u_{m-k}^{n+1}}
\end{aligned}
$$

Since (47) with $k$ has been proved and we can use (48) for $j=k-1$, we see that (48) holds for $j=k$. (This argument is invalid if $\alpha=1 / k$, since the right hand side of (50) is zero in the case.)

Before proving (49) with $j=k$, we claim that $u_{m-k-1}^{n+1}$ is bounded in $n$ if $\alpha>1 /(k+1)$. In fact, we have by $(32)$

$$
\begin{aligned}
u_{m-k-1}^{n+1} & \leq \lambda_{n} u_{m-k}^{n}+\left\{1+\Delta t_{n}\left(u_{m-k-1}^{n}\right)^{\alpha}\right\} u_{m-k-1}^{n} \\
& \leq \lambda_{n} u_{m-k}^{n}+\left\{1+\tau b_{n} a_{n}^{\alpha}\right\} u_{m-k-1}^{n} \\
& \leq \lambda_{n} u_{m-k}^{n}+\left\{1+\tau h^{-\alpha / p} a_{n}^{\alpha}\right\} u_{m-k-1}^{n} \equiv B_{n}+A_{n} u_{m-k-1}^{n}
\end{aligned}
$$


By (47), we have

$$
\frac{B_{n+1}}{B_{n}}=\left(\frac{u_{m}^{n}}{u_{m}^{n+1}}\right)^{\alpha} \frac{u_{m-k}^{n+1}}{u_{m-k}^{n}}=\left(1+\tau h^{-\alpha / p}\right)^{-\alpha-\min \{1, k \alpha\}+1}<1 \quad \text { for } \quad \alpha>\frac{1}{k+1},
$$

and thus $\sum_{n=0}^{\infty} B_{n}<\infty$. On the other hand, by the same argument as is used in the proof of Lemma 4.6, we have $\prod_{n=0}^{\infty} A_{n}<\infty$. Hence, by Lemma 4.7, we see that $\left\{u_{m-k-1}^{n+1}\right\}_{n=0}^{\infty}$ is bounded if $\alpha>1 /(k+1)$.

As for (49), it is trivial in the case of $1 /(k+1)<\alpha \leq 1 / k$ since $u_{m-k}^{n}$ tends to infinity while $u_{m-k-1}^{n}$ remains bounded as $n$ tends to infinity. If $\alpha \leq 1 /(k+1)$, we have by $(32)$

$$
\begin{aligned}
\frac{u_{m-k-1}^{n+1}}{u_{m-k}^{n+1}} & \leq \lambda_{n} \frac{u_{m-k}^{n}}{u_{m-k}^{n+1}}+\left\{1+\Delta t_{n}\left(u_{m-k-1}^{n}\right)^{\alpha}\right\} \frac{u_{m-k-1}^{n}}{u_{m-k}^{n}} \cdot \frac{u_{m-k}^{n}}{u_{m-k}^{n+1}} \\
& \leq\left[\lambda_{n}+\left(1+\tau b_{n} a_{n}^{\alpha}\right) \frac{u_{m-k-1}^{n}}{u_{m-k}^{n}}\right] \cdot \frac{u_{m-k}^{n}}{u_{m-k}^{n+1}},
\end{aligned}
$$

and then (47) with $j=k$ yields that

$$
\limsup _{n \rightarrow \infty} \frac{u_{m-k-1}^{n+1}}{u_{m-k}^{n+1}} \leq\left(1+\tau h^{-\alpha / p}\right)^{k \alpha-1} \limsup _{n \rightarrow \infty} \frac{u_{m-k-1}^{n}}{u_{m-k}^{n}} .
$$

This implies that $\lim \sup _{n \rightarrow \infty} u_{m-k-1}^{n} / u_{m-k}^{n}=0$. Namely, (49) holds ford $k$.

REMARK 4.4. Theorem 4.2 says in some sense that the numerical blow-up set does not precisely correspond to the blow-up set of original PDE. However, note that as $h \rightarrow 0$ all the numerical blow-up points tends to $x=1 / 2$. It simply shows that a single experiment is not sufficient to see quantitative results. So, this is not against a common sense of numerical computations. The example in the next section is, however, somewhat deeper.

\section{Asymptotic behavior in the case of $f(u)=u[\log (1+u)]^{\beta}$}

In this section, we consider equation (13) with nonlinear term $f(u)=u[\log (1+$ $u)]^{\beta}$. Since no blow-up occurs if $\beta \leq 1$, we assume that $1<\beta$. The difference scheme is rewritten as

$$
\frac{u_{j}^{n+1}-u_{j}^{n}}{\Delta t_{n}}=\frac{u_{j+1}^{n}-2 u_{j}^{n}+u_{j-1}^{n}}{h^{2}}+u_{j}^{n}\left[\log \left(1+u_{j}^{n}\right)\right]^{\beta},
$$

$\Delta t_{n}$ is now defined as

$$
\Delta t_{n}=\tau \min \left\{1, \frac{1}{\left[\log \left(1+\left\|u^{n}\right\|_{p}\right)\right]^{\beta}}\right\} .
$$

We are going to show that the scheme (51) results in a curious asymptotic behavior, which is essentially different from those in the previous section. Friedman 
and McLeod [8] considered the PDE with $f(u)=u[\log (1+u)]^{\beta}$ in the case of $\beta>2$, and they found that its blow-up set consisted, under a certain assumption, only of one point $x=1 / 2$. Later Lacey [11] studied the case of $1<\beta \leq 2$, and showed that its blow-up set was the whole interval $(0,1)$ if $\beta<2$. We study the numerical blow-up set of (51) and compare it with the blow-up set of PDE.

THEOREM 5.1. Suppose that $\beta>1$. Let $\left\{u_{j}^{n}\right\}$ be a solution of (51) with the boundary condition (17) and an appropriate initial data such that $\left\{u_{j}^{n}\right\}$ blows up. Assume that (A1) and (A2) hold. Then for all $j=1,2, \ldots, N-1$, it holds that

$$
\lim _{n \rightarrow \infty} u_{j}^{n}=\infty .
$$

Note that the conclusion is true for all $1<\beta<\infty$.

For the proof of Theorem 5.1, we prove two limit relations:

$$
\begin{aligned}
& \lim _{n \rightarrow \infty} \frac{\log \left(1+u_{m}^{n}\right)}{\log \left(1+\left\|u^{n}\right\|_{p}\right)}=1, \\
& \lim _{n \rightarrow \infty} \frac{u_{m}^{n}}{u_{m}^{n+1}}=\frac{1}{1+\tau} .
\end{aligned}
$$

To prove (52), we note that $\left\|u^{n}\right\|_{p} \leq\left\|u^{n}\right\|_{\infty} \leq h^{-1 / p}\left\|u^{n}\right\|_{p}$. Since $\left\|u^{n}\right\|_{\infty}=u_{m}^{n}$, we have

$$
1 \leq \frac{\log \left(1+u_{m}^{n}\right)}{\log \left(1+\left\|u^{n}\right\|_{p}\right)} \leq \frac{\log h^{-1 / p}}{\log \left(1+\left\|u^{n}\right\|_{p}\right)}+1 .
$$

By letting $n \rightarrow \infty$, we obtain (52).

We next note that for sufficiently large $n$

$$
\frac{u_{m}^{n+1}}{u_{m}^{n}}=2 \lambda_{n} \frac{u_{m-1}^{n}}{u_{m}^{n}}+1-2 \lambda_{n}+\tau\left[\frac{\log \left(1+u_{m}^{n}\right)}{\log \left(1+\left\|u^{n}\right\|_{p}\right)}\right]^{\beta} .
$$

This and (52) yields (53).

The relation (53) shows that $c\left(1+u_{m}^{n}\right) \leq 1+u_{m}^{n+1} \leq C\left(1+u_{m}^{n}\right)$, where $c$ and $C$ are independent of $n$. This implies that

$$
\lim _{n \rightarrow \infty} \frac{\log \left(1+u_{m}^{n+1}\right)}{\log \left(1+u_{m}^{n}\right)}=1 .
$$

We are now in a position to prove Theorem 5.1.

Proof of Theorem 5.1. We show inductively in $j=m-1, \ldots, 1$ that

$$
\begin{aligned}
& \lim _{n \rightarrow \infty} u_{j}^{n}=\infty, \\
& \lim _{n \rightarrow \infty} \frac{\log \left(1+u_{j}^{n+1}\right)}{\log \left(1+u_{j}^{n}\right)}=1, \\
& \lim _{n \rightarrow \infty} \frac{\log \left(1+u_{j}^{n}\right)}{\log \left(1+u_{j+1}^{n}\right)}=1 .
\end{aligned}
$$


This is sufficient for the proof.

We first prove (58) for $j=m-1$. The equation (51) shows that $u_{m-1}^{n+1} \geq \lambda_{n} u_{m}^{n}$ for all sufficiently large $n$. Therefore $1+u_{m-1}^{n+1} \geq \lambda_{n}\left(1+u_{m}^{n}\right)$. Consequently we obtain

$$
\begin{aligned}
\log \left(1+u_{m-1}^{n+1}\right) & \geq \log \lambda_{n}+\log \left(1+u_{m}^{n}\right) \\
& \geq \log \lambda-\beta \log \left\{\log \left(1+\left\|u^{n}\right\|_{p}\right)\right\}+\log \left(1+u_{m}^{n}\right)
\end{aligned}
$$

Further, by (52) and (55), we have

$$
\begin{aligned}
1 & \geq \frac{\log \left(1+u_{m-1}^{n+1}\right)}{\log \left(1+u_{m}^{n+1}\right)} \\
& \geq \frac{\log \lambda}{\log \left(1+u_{m}^{n+1}\right)}-\beta \cdot \frac{\log \left(1+\left\|u^{n}\right\|_{p}\right)}{\log \left(1+u_{m}^{n+1}\right)} \cdot \frac{\log \left\{\log \left(1+\left\|u^{n}\right\|_{p}\right)\right\}}{\log \left(1+\left\|u^{n}\right\|_{p}\right)}+\frac{\log \left(1+u_{m}^{n}\right)}{\log \left(1+u_{m}^{n+1}\right)} \\
& \rightarrow 1 \text { as } n \rightarrow \infty .
\end{aligned}
$$

Thus we have (58) for $j=m-1$. Since $\lim _{n \rightarrow \infty} u_{m}^{n}=\infty$, (58) for $j=m-1$ implies (56) for $j=m-1$.

To prove (57) for $j=m-1$, note that

$$
\frac{\log \left(1+u_{m-1}^{n+1}\right)}{\log \left(1+u_{m-1}^{n}\right)}=\frac{\log \left(1+u_{m-1}^{n+1}\right)}{\log \left(1+u_{m}^{n+1}\right)} \frac{\log \left(1+u_{m}^{n+1}\right)}{\log \left(1+u_{m}^{n}\right)} \frac{\log \left(1+u_{m}^{n}\right)}{\log \left(1+u_{m-1}^{n}\right)} .
$$

Since we already know that each fraction of the right hand side tends to unity, we have proved (57) for $j=m-1$. Thus we have shown (56)-(58) for $j=m-1$.

For $j<m-1$, we proceed quite similarly. Suppose that (56)-(58) holds true for $j+1$. Then note that $u_{j}^{n+1} \geq \lambda_{n} u_{j+1}^{n}$ for sufficiently large $n$, which yields that

$$
\frac{\log \left(1+u_{j}^{n+1}\right)}{\log \left(1+u_{j+1}^{n+1}\right)} \geq \frac{\log \lambda}{\log \left(1+u_{j+1}^{n+1}\right)}-\beta \frac{\log \left\{\log \left(1+\left\|u^{n}\right\|_{p}\right)\right\}}{\log \left(1+u_{j+1}^{n+1}\right)}+\frac{\log \left(1+u_{j+1}^{n}\right)}{\log \left(1+u_{j+1}^{n+1}\right)} .
$$

The first and last terms of the right hand side tend to zero and unity, respectively, by the assumption of the induction. The second term is:

$$
\frac{\log \left\{\log \left(1+\left\|u^{n}\right\|_{p}\right)\right\}}{\log \left(1+u_{j+1}^{n+1}\right)}=\frac{\log \left(1+u_{j+2}^{n+1}\right)}{\log \left(1+u_{j+1}^{n+1}\right)} \frac{\log \left(1+u_{j+3}^{n+1}\right)}{\log \left(1+u_{j+2}^{n+1}\right)} \cdots \frac{\log \left\{\log \left(1+\left\|u^{n}\right\|_{p}\right)\right\}}{\log \left(1+u_{m}^{n+1}\right)} .
$$

All the terms except for the last tends to unity, and the last tends to zero. We have therefore proved (58) for $j$. From this immediately follows (56) for $j$. As for (57), simply note that

$$
\frac{\log \left(1+u_{j}^{n+1}\right)}{\log \left(1+u_{j}^{n}\right)}=\frac{\log \left(1+u_{j}^{n+1}\right)}{\log \left(1+u_{j+1}^{n+1}\right)} \frac{\log \left(1+u_{j+1}^{n+1}\right)}{\log \left(1+u_{j+1}^{n}\right)} \frac{\log \left(1+u_{j+1}^{n}\right)}{\log \left(1+u_{j}^{n}\right)},
$$


the right hand side of which tends to one.

REMARK 5.1. Theorem 5.1 shows that in the case of $\beta>2$, the numerical blow-up set cannot faithfully reproduce that of the corresponding PDE. It suggests that with 'weak' nonlinearity, the asymptotic behavior of the numerical solution (with uniform spatial grids) near the blow-up time cannot simulate the asymptotic behavior of the solution of PDE.

\section{Concluding remarks}

Though we believe that we have made a substantial progress, we are unable to answer three questions. The first one is: In the case of $f(u)=u^{1+\alpha}$ we proved that $\lim _{n \rightarrow \infty} u_{m-1}^{n} / u_{m}^{n}=0$. We expect, based on our numerical experiments, that the same is true in the case of $f(u)=u[\log (1+u)]^{\beta}$, too. We are, however, unable to prove this. The second, more important question is to derive a convergence rate for $|T(t, h)-T|$ in the case of PDE. This seems to require inequalities more accurate than those used in the present paper. Finally, when $f(u)=u^{1+\alpha}$ and $g(s)=s^{\gamma}$ in Section 3, we would like to determine the best choice of $\gamma$. These questions are left to the future study.

Acknowledgment. The present paper is an outgrowth of the first author's master's thesis [5] under the guidance of the third author in RIMS, Kyoto University. Hospitality of RIMS to him is highly acknowledged. His stay in Kyoto University was supported by the Panasonic Scholarship, to which he is very grateful. The third author is partially supported by the Grant-in-Aid of JSPS, No. 17204008

\section{References}

[1] L. Abia, J.C. López-Marcos and J. Martínez, Blow-up for semidiscretizations of reactiondiffusion equations. Appl. Numer. Math., 20 (1996), 145-156.

[ 2 ] L. Abia, J.C. López-Marcos and J. Martínez, On the blow-up time convergence of semidiscretizations of reaction-diffusion equations. Appl. Numer. Math., 26 (1998), 399-414.

[ 3 ] L.M. Abia, J.C. López-Marcos and J. Martínez, The Euler method in the numerical integration of reaction-diffusion problems with blow-up. Appl. Numer. Math., 38 (2001), 287-313.

[ 4 ] L.A. Caffarrelli and A. Friedman, Blow-up of solutions of nonlinear heat equations. J. Math. Anal. Appl., 129 (1988), 409-419.

[ 5 ] C.-H. Cho, On a finite difference scheme for the parabolic blow-up problems. Master's thesis, Research Institute for Mathematical Sciecnes, Kyoto University, 2005.

[6] Y.-G. Chen, Asymptotic behaviours of blowing-up solutions for finite difference analogue of $u_{t}=u_{x x}+u^{1+\alpha}$. J. Fac. Sci., Univ. Tokyo, 33 (1986), 541-574.

[ 7 ] P.J. Davis and P. Rabinowitz, Methods of Numerical Integration. Academis Press, 1975.

[8] A. Friedman and B. McLeod, Blow-up of positive solutions of semilinear heat equations. Indiana Univ. Math. J., 34 (1985), 425-447.

[9] C. Hirota and K. Ozawa, A methof of estimating the blow-up time and blow-up rate of the solution of the system of ordinary sifferencial equations - An application to the blow-up problems of partial differencial equations-(in Japanese). Trans. Japan SIAM, 14 (2004), 13-38.

[10] S. Itô, On blow-up of positive solutions of semilinear parabolic equations. J. Fac. Sci. Univ. Tokyo, Sect. IA, 37 (1990), 527-536. 
[11] A.A. Lacey, Global blow-up of a nonlinear heat equation. Proc. R. Soc. Edin., 104 A (1986), 161-167.

[12] T. Nakagawa, Blowing up of a finite difference solution to $u_{t}=u_{x x}+u^{2}$. Appl. Math. Optim., 2 (1976), 337-350.

[13] T. Nakagawa and T. Ushijima, Finite element analysis of the semi-linear heat equation of blow-up type. in Topics in Numer. Anal. III, ed. J.J.H. Miller, 1977, 275-291.

[14] W. Ren and X.-P. Wang, An iterative grid redistribution method for singular problems in multiple dimensions. J. Comp. Phys., 159 (2000), 246-273.

[15] M. Tabata, A finite difference approach to the number of peaks of solutions for semilinear parabolic problems. J. Math. Soc. Japan, 32 (1980), 171-191.

[16] T.K. Ushijima, On the approximation of blow-up time for solutions of nonlinear parabolic equations. Publ. RIMS, 36 (2000), 613-640.

[17] F. Weissler, Single point blowup of semilinear initial value problems. J. Diff. Eqns., 55 (1984), 202-224. 